J. Nonlinear Var. Anal. 2 (2018), No. 3, pp. 355-378

Available online at http://jnva.biemdas.com

https://doi.org/10.23952/jnva.2.2018.3.09

\title{
SUBDIFFERENTIALS AND SNC PROPERTY OF SCALARIZATION FUNCTIONALS WITH UNIFORM LEVEL SETS AND APPLICATIONS
}

\author{
TRUONG Q. BAO ${ }^{1}$, CHRISTIANE TAMMER ${ }^{2, *}$ \\ ${ }^{1}$ Department of Mathematics and Computer Science, Northern Michigan University, \\ 1401 Presque Isle Avenue, Marquette, MI 49855, USA \\ ${ }^{2}$ Institute of Mathematics, Martin-Luther-University Halle-Wittenberg, Theodor-Lieser Str. 5, 06120 Halle, Germany
}

\begin{abstract}
This paper deals with necessary conditions for minimal solutions of constrained and unconstrained optimization problems with respect to general domination sets by using the well-known nonlinear scalarization functional with uniform level sets (called Gerstewitz' functional in the literature). The primary objective of this paper is to establish revised formulas for basic and singular subdifferentials of these nonlinear scalarization functionals and study important properties such as the PSNC property, the Lipschitz behavior, etc. of these scalarization functionals without assuming that the shifted set involved in the definition of the functional is convex. The second objective is to propose a new way to scalarize a set-valued optimization problem. It allows us to study necessary conditions for minimal solutions in a very broad setting in which the domination set is not necessarily convex or solid or conical. The third objective is to apply our results to vector-valued approximation problems. Keywords. Domination sets; Minimal solutions; Optimization problems; Scalarization functionals; Subdifferentials.
\end{abstract}

2010 Mathematics Subject Classification. 90C29, 90C26, 58C07, 90C48.

\section{INTRODUCTION}

In this paper, we provide revised formulas for subdifferentials of a nonlinear scalarization functional with uniform level sets (called Gerstewitz' functional in the literature) and study important properties such as the PSNC property, the Lipschitz behavior, etc. of these scalarization functionals without assuming that the shifted set involved in the definition of the functional is convex. This allows us to establish necessary conditions for minimal solutions of constrained and unconstrained optimization problems with respect to general domination sets by using a nonlinear scalarization approach.

In vector optimization theory, one works with convex ordering cones which induce order relations on the underlying spaces. Recently, there has been a growing interest in vector optimization with domination sets; in particular, in [22], Tammer introduced a new $\varepsilon$-solution concept for domination sets along a scalarization direction and presented a vector-valued variational principle for this new type of approximation solutions. In [21, Definition 5.53], Mordukhovich defined an abstract concept of optimality that covers conventional notions of optimal solutions to multiobjective problems and that is induced by the concept of set extremality. In [2, Chapter 5], Bao and Mordukhovich established necessary conditions

\footnotetext{
${ }^{*}$ Corresponding author.

E-mail addresses: btruong@nmu.edu (T.Q. Bao), christiane.tammer@mathematik.uni-halle.de (Chr. Tammer).

Received August 16, 2018; Accepted October 12, 2018.
}

(C)2018 Journal of Nonlinear and Variational Analysis 
for minimizers of vector-valued optimization problems with respect to domination sets enjoying the socalled asymptotic closedness property by using a dual space (known also as variational) approach. In [13], Huang, Rubinov and Yang considered problems of vector optimization with nonconvex preferences which are generated by a union of a finite number of convex and solid cones.

Scalarization is an important tool in the study of vector optimization in which solutions of a multiobjective optimization problem could be studied and found by solving a single-objective optimization problem. Note that the majority of publications on vector optimization problems in the scalarization approach need the nonempty interiority condition imposed on the ordering cone. This requirement is strict in infinite-dimensional spaces; outside the finite dimensional case there exist only a few examples when the natural ordering cone has a nonempty interior. Recently, a few recent papers seem to work on Pareto minimal solution of vector optimization in general Banach spaces, but they indeed dealt with certain kinds of proper efficient solutions since one could construct a solid ordering cone such that a Pareto minimal solution under consideration is also efficient with respect to the solid constructed cone. For example, in [7], under assumptions made by Durea and Dutta we could construct a solid Henig dilating cone and in [3] Bao and Tammer proposed to use a smaller solid cone that could be viewed as a component of a solid Henig dilating cone generated by a nonzero vector in the given ordering cone.

It is important to emphasize that we investigate the necessary optimality conditions for set-valued optimization problems based on the vector approach, i.e., for solutions defined on the graph of the set-valued objective map (see [17]) and that they are new even in the case of vector optimization with single-valued cost functions. Our technique bases on new developments of calculus rules for generalized differentiation and a new way to scalarize a set-valued optimization problem into a scalar one whose cost function is not a composition of the scalarization functional and the given cost mapping; a composition rule for set-valued mappings is too complicated to manipulate without certain Lipschitz continuity requirements.

Our paper is organized as follows: Section 2 recalls the scalarization technique by means of nonlinear functionals with uniform level sets and the concepts of generalized differentiation by Mordukhovich $[20,21]$. In Section 3, we derive formulas for both basic and singular subdifferentials of the scalarization functional with uniform level sets where the shifted set is proper and closed, but not necessarily convex. In Section 4, we show the necessary optimality conditions for set-valued optimization problems based on the vector approach. Finally, we apply our results to vector-valued approximation problems in Section 5 and give some conclusions in Section 6.

\section{PRELIMINARIES}

2.1. Scalarization approach in vector optimization. Let $Y$ be a real linear topological space, $A$ be a nonempty set in $Y, \mathbb{R}_{+}$be the set of all nonnegative numbers. The notation int $A, \operatorname{cl} A$ and $\operatorname{bd} A$ stand for the topological interior, closure and boundary of the set $A$, respectively. $A$ is said to be solid if int $A \neq \emptyset$, proper if $A \neq \emptyset$ and $A \neq Y$, pointed if $A \cap(-A) \subseteq\{\mathbf{0}\}$, a cone if $\forall a \in A, \forall t \in \mathbb{R}_{+}:$ta $\in A$, and a convex cone if $A$ is a cone and $A+A=A$. A proper cone with $A \neq\{0\}$ is called nontrivial.

The recession cone of $A$ is defined by

$$
A_{\infty}:=\left\{y \in Y \mid \forall a \in A, \forall t \in \mathbb{R}_{+}: a+t y \in A\right\} .
$$

See $[15,16]$ for basic definitions and concepts of vector optimization. 
Let $Y$ be equipped with a binary relation generated by a domination set $\Theta \subset Y$ being proper and $0 \in \operatorname{cl} \Theta$. Denoting the relation on $Y$ with respect to $\Theta$ by " $\leq{ }_{\Theta}$ ", we have:

$$
y_{1} \leq_{\Theta} y_{2}: \Longleftrightarrow y_{1} \in y_{2}-\Theta .
$$

When $\Theta=C$ is a nontrivial, closed, convex and pointed cone, $\leq_{C}$ is a partial order in $Y$. In this paper, we do not impose either the convexity property or the conical property for the domination set $\Theta$.

Extending the classical Pareto minimality notion in vector optimality from ordering cones to domination sets, we say that a point $\bar{y} \in \Xi$, where $\Xi \subseteq Y$ is a nonempty subset in $Y$, is a $\Theta$-minimal point of $\Xi$, known also as a $\Theta$-efficient point of $\Xi$ or a minimal point of $\Xi$ with respect to the domination set $\Theta$, if

$$
\Xi \cap(\bar{y}-\Theta)=\{\bar{y}\}
$$

i.e., for every $y \in \Xi, y \leq_{\Theta} \bar{y}$ implies $y=\bar{y}$.

In vector optimization, scalarization is of great importance. It allows us to convert a vector optimization problem into a suitable scalar optimization problem which is an optimization problem with a real-valued objective functional; and thus we could use the well-developed scalar optimization theory. In this paper, we use Gerstewitz' nonlinear scalarization functional since its subdifferentials could be fully computed; see Section 3.

Let us recall this nonlinear scalarization functional and several important properties in [14, 22]; see also $[15,24]$.

Definition 2.1. (scalarization directions of sets). Let $A$ be a proper subset in a linear space $Y$. A nonzero vector $\mathbf{k} \in Y$ is called a SCALARIZATION DIRECTION of $A$ if $A$ does not contain lines parallel to $\mathbf{k}$ and the condition

$$
\forall t \in \mathbb{R}_{+}: A+t \mathbf{k} \subseteq A
$$

holds. The set of all scalarization directions of $A$ is denoted by $\operatorname{dir}(A)$.

Definition 2.2. (nonlinear scalarization functionals). Let $A$ be a proper set in a linear space $Y$ and $\mathbf{k} \in \operatorname{dir}(A)$ be a scalarization direction of $A$. The functional $\varphi_{A, \mathbf{k}}: Y \rightarrow \mathbb{R} \cup\{+\infty\}$ defined by

$$
\varphi_{A, \mathbf{k}}(y):=\inf \{t \in \mathbb{R} \mid y \in t \mathbf{k}-A\},
$$

where $\inf (\emptyset)=+\infty$, is called nonlinear scalarization functional with uniform level sets in respect of the set $A$ and the scalarization direction $\mathbf{k}$. For the sake of simplicity, we will call it a scalarization functional.

In the literature, the functional (2.4) is called Gerstewitz' functional. This functional is intensively studied by Gerth and Weidner in [14].

Note that $\operatorname{dir}(C)=C \backslash(-C)$ for any closed and convex cone $C$. Note also that if $A$ contains a line parallel to $k$; i.e., there exists an element $\bar{y} \in A$ such that $\bar{y}+t \mathbf{k} \in A$ for all $t \in \mathbb{R}$, then $\varphi_{A, \mathbf{k}}(\bar{y})=-\infty$. The scalarization direction requirement $\mathbf{k} \in \operatorname{dir}(\Theta)$ ensures that $\varphi_{\Theta, \mathbf{k}}$ takes values in $\mathbb{R} \cup\{+\infty\}$. In [24], Tammer and Zălinescu studied the scalarization functional (2.4) for the class of sets enjoying the freedisposal propery, i.e., $A-K=A$ for some proper, closed and convex cone $K$. In such a situation, the cone $-A_{\infty}$ is the biggest closed and convex cone $K$ verifying the free-disposal property $A-K=A$ and a scalarization direction satisfies $\mathbf{k} \in K \backslash(-K)$ and $\mathbf{k} \in-A_{\infty}$.

The following lemmas provide several known properties of Gersterwitz' scalarization functionals. 
Lemma 2.1. ([15, Theorem 2.3.1]) Consider the scalarization functional $\varphi_{A, \mathbf{k}}$ introduced in Definition 2.2. Assume that $A$ is a closed set in a real topological linear space $Y$. Then, the following assertions hold:

(a) $\varphi_{A, \mathbf{k}}$ is lower semicontinuous over its domain $\operatorname{dom}\left(\varphi_{A, \mathbf{k}}\right)=\mathbb{R} \mathbf{k}-$ A. For every real number $\tau \in \mathbb{R}$, the $\tau$-level set of $\varphi_{A, \mathbf{k}}$ is given by

$$
\operatorname{Lev}\left(\tau ; \varphi_{A, \mathbf{k}}\right):=\left\{y \in Y \mid \varphi_{A, \mathbf{k}}(y) \leq \tau\right\}=\tau \mathbf{k}-A .
$$

(b) $\varphi_{A, \mathbf{k}}$ is linearly shifted along the scalarization direction $\mathbf{k}$ in the sense that

$$
\forall y \in Y, \forall t \in \mathbb{R}: \varphi_{A, \mathbf{k}}(y+t \mathbf{k})=\varphi_{A, \mathbf{k}}(y)+t .
$$

(c) $\varphi_{A, \mathbf{k}}$ is convex if and only if $A$ is convex, and positively homogeneous if and only if $A$ is a cone.

Remark 2.1. (on the closedness of $A$ ). We always have $\varphi_{A, \mathbf{k}}=\varphi_{\mathrm{vcl}_{\mathbf{k}}(A), \mathbf{k}}$ for any set $A$ in $Y$, where $\operatorname{vcl}_{\mathbf{k}}(A)$ is defined by

$$
\operatorname{vcl}_{\mathbf{k}}(A):=\{y \in Y \mid \forall \tau>0, \exists t \in[0, \tau]: y+t \mathbf{k} \in A\}
$$

and is called the vector closure of $A$ in the direction $\mathbf{k}$. If $A$ is a closed set, then $A=\operatorname{cl}(A)=\operatorname{vcl}_{\mathbf{k}}(A)$. However, the reverse is not true. For example, let

$$
A:=\left\{(a, b) \in \mathbb{R}^{2} \mid \forall a \in(0,-1): b=-\sqrt{1-a^{2}}\right\}
$$

be a set and $\mathbf{k}=(1,1)$ be a direction in $\mathbb{R}^{2}$. We have $A=\operatorname{vcl}_{\mathbf{k}}(A)$ but $\operatorname{cl} A=A \cup\{(-1,0),(0,-1)\}$.

In the classical setting of vector optimization, where the domination set $\Theta=C$ is assumed to be a nontrivial, closed, solid and convex cone, the scalarization functional $\varphi_{C, \mathbf{k}}$ with $\mathbf{k} \in \operatorname{int}(C)$ has many good properties. In addition, the exact forms of subdifferentials of the scalarization functional $\varphi_{C, \mathbf{k}}$ are computed via the positive polar cone of $C$ denoted by

$$
C^{+}:=\left\{y^{*} \in Y^{*} \mid \forall y \in C: y^{*}(y) \geq 0\right\} .
$$

Lemma 2.2. ([9, Lemma 2.1]). Let $Y$ be a real topological linear space, $C$ be a nontrivial, closed, solid and convex cone in $Y$, and $\mathbf{k} \in \operatorname{int}(C)$ be a scalarization direction of $C$. The scalarization functional $\varphi_{C, \mathbf{k}}$ defined in Definition 2.2 is continuous, sublinear, int $(C)$-monotone, and linearly shifted along the direction $\mathbf{k}$. For every $\bar{y} \in Y$, the subdifferential of $\varphi_{C, \mathbf{k}}$ at $\bar{y} \in Y$ is given by

$$
\partial \varphi_{C, \mathbf{k}}(\bar{y})=\left\{y^{*} \in C^{+} \mid y^{*}(\mathbf{k})=1 \wedge y^{*}(\bar{y})=\varphi_{C, \mathbf{k}}(\bar{y})\right\}
$$

where $C^{+}$is the positive polar cone of $C$. When $\bar{y}=\mathbf{0}$, (2.5) becomes

$$
\partial \varphi_{C, \mathbf{k}}(\mathbf{0})=C^{+} \cap H_{1}(\mathbf{k}) \text { with } H_{1}(\mathbf{k}):=\left\{y^{*} \in Y^{*} \mid y^{*}(\mathbf{k})=1\right\} .
$$

Lemma 2.3. ([9, Theorem 2.2]) Let $Y$ be a real topological linear space, and let $A$ be a nontrivial, closed and convex set in $Y$, and $\mathbf{k}$ be a scalarization direction of $A$. Then, for every $\bar{y} \in \operatorname{dom} \varphi_{A, \mathbf{k}}$, the subdifferential (of convex analysis) of the scalarization functional $\varphi_{A, \mathbf{k}}$ at $\bar{y}$ is given by

$$
\partial \varphi_{A, \mathbf{k}}(\bar{y})=\left\{y^{*} \in Y^{*} \mid y^{*}(\mathbf{k})=1 \wedge \forall y \in A: y^{*}(\bar{y}-y)-\varphi_{A, \mathbf{k}}(\bar{y}) \geq 0\right\} .
$$


Lemma 2.4. ([24, Corollary 4.2]) Let $Y$ be a separated locally convex space, $A \subseteq Y$ be a nontrivial, closed and convex set enjoying the free-disposal property, and $\mathbf{k} \notin A_{\infty}$. Then for every $\bar{y} \in Y$ the subdifferential (of convex analysis) of the scalarization functional $\varphi_{A, \mathbf{k}}$ at $\bar{y}$ is given by

$$
\partial \varphi_{A, \mathbf{k}}(\bar{y})=\left\{y^{*} \in \operatorname{bar} A \mid y^{*}(\mathbf{k})=1 \wedge \forall y \in A: y^{*}(\bar{y}-y)-\varphi_{A, \mathbf{k}}(\bar{y}) \geq 0\right\},
$$

where bar $A$ is the domain of the support function or the barrier cone of $A$.

It is important to emphasize that since we do not assume that $A$ is convex in this paper, the scalarization functional defined in Definition 2.2 might not convex and thus we could not use generalized differentiation in convex analysis. We need to use Mordukhovich/limiting generalized differentiatial constructions which has a rich calculus in the Asplund setting. Observe that when $A$ is nonsolid, i.e., int $A=\emptyset$, the scalarization functional $\varphi_{A, \mathbf{k}}$ is not Lipschitz, the so-called singular subdifferential plays an important role for the validity of calculus rules. Note finally that one could establish the corresponding results in terms of other generalized differentiation; for example, the Ioffe approximation subdifferential and the Rockafellar-Clarke convex subdifferential in the more general Banach setting.

2.2. Mordukhovich/limiting generalized differentiation. Throughout the paper, we use the standard notation of variational analysis; cf. [20,21], and assume that all the spaces under consideration are Asplund unless otherwise stated. Recall that a Banach space is Asplund if every convex continuous functional $\varphi: U \rightarrow \mathbb{R}$ defined on an open convex subset $U$ of $X$ is Fréchet/regular differentiable on a dense subset of $U$. The class of Asplund spaces is quite broad including every reflexive Banach space and every Banach space with a separable dual; in particular, $c_{0}$ and $\ell_{p}, L_{p}[0,1]$ for $1<p<\infty$ are Asplund spaces. In this section, we present the definitions and properties of the Mordukhovich/limiting differential constructions in the Asplund space setting. For their useful modifications in general Banach spaces, see $[20,21]$ and the bibliographies therein.

Given an Asplund space $X$, we denote its norm by $\|\cdot\|$ and consider the dual space $X^{*}$ equipped with the weak* topology $w^{*}$, where $x^{*}(x)$ stands for the canonical pairing between $X^{*}$ and $X$. Given a nonempty set $\Omega$ in $X$ and $\bar{x} \in \Omega$. We say that $\Omega$ is locally closed around $\bar{x}$ if there is a neighborhood $U$ of $\bar{x}$ such that $\Omega \cap \mathrm{cl} U$ is a closed set.

We use the capital letters for set-valued mappings, the lower case letters for single-valued functionals, and the Greek letters for extended-real-valued functionals. Given a set-valued mapping $F: X \rightrightarrows Y$ between two Asplund spaces. Denote by $\operatorname{dom} F:=\{x \in X \mid F(x) \neq \emptyset\}, \operatorname{gph} F:=\{(x, y) \in X \times Y \mid y \in$ $F(x)\}$, and $F(\Omega):=\cup\{F(x) \mid x \in \Omega\}$ the domain, the graph, and the image set of $F$ over $\Omega$, respectively. A single-valued function $f: X \rightarrow Y$ can be viewed as a special form of the following set-valued mapping $F$ with

$$
F(x):= \begin{cases}\{f(x)\}, & \text { if } x \in \operatorname{dom} f \\ \emptyset, & \text { if } x \notin \operatorname{dom} f\end{cases}
$$

For the sake of simplicity, we will write $F(x)=f(x)$ and the sum of a set-valued mapping $F$ and a single-valued function $g$ is expressed by

$$
(F+g)(x)=F(x)+g(x) .
$$

The generalized composition between a set-valued mapping $F: X \rightrightarrows Y$ and a functional $\varphi: Y \rightrightarrows \mathbb{R}$ is a scalar set-valued mapping $\varphi \circ F$ acting from $X$ into $\mathbb{R}$ with images $\varphi \circ F(x):=\cup\{\varphi(y) \in \mathbb{R} \mid y \in F(x)\}$. 
Definition 2.3. (normal cones). Let $\Omega$ be a nonempty set in an Asplund space $X$.

(i) The REgUlar NORMAL CONE (known also as the prenormal or Fréchet normal cone) to $\Omega$ at $x \in \Omega$ is defined by

$$
\widehat{N}(x ; \Omega):=\left\{x^{*} \in X^{*} \mid \underset{u \stackrel{\Omega}{\rightarrow}_{x}}{\limsup } \frac{x^{*}(u-x)}{\|u-x\|} \leq 0\right\} .
$$

(ii) Assume that $\Omega$ is locally closed around $\bar{x} \in \Omega$. The LIMITING NORMAL CONE (known also as the basic or Mordukhovich normal cone) to $\Omega$ at $\bar{x}$ is defined by

$$
\begin{aligned}
N(\bar{x} ; \Omega) & :=\operatorname{Limsup}_{x \rightarrow \bar{x}} \widehat{N}(x ; \Omega) \\
& =\left\{x^{*} \in X^{*} \mid \exists x_{k} \rightarrow \bar{x}, x_{k}^{*} \stackrel{w^{*}}{\rightarrow} x^{*} \text { with } x_{k}^{*} \in \widehat{N}\left(x_{k} ; \Omega\right)\right\},
\end{aligned}
$$

where Lim sup stands for the sequential Painlevé-Kuratowski outer limit of regular normal cones to $\Omega$ at $x$ as $x$ tends to $\bar{x}$.

It is known that the Mordukhovich normal cone (2.9) being nonconvex in general enjoys a rich calculus in Asplund spaces in comparison with the convex regular normal cone (2.8), and that both cones (2.9) and (2.8) reduce to the normal cone of convex analysis when $\Omega$ is convex.

Definition 2.4. (coderivatives of set-valued mappings). Let $F: X \rightrightarrows Y$ be a set-valued mapping between Asplund spaces. Assume that $\operatorname{gph} F$ is locally closed around $(\bar{x}, \bar{y}) \in \operatorname{gph} F$.

(i) The Regular Coderivative $\widehat{D}^{*} F(\bar{x}, \bar{y}): Y^{*} \rightrightarrows X^{*}$ of $F$ at $(\bar{x}, \bar{y})$ is defined via the normal cone to $\operatorname{gph} F$ at $(\bar{x}, \bar{y})$ by

$$
\widehat{D}^{*} F(\bar{x}, \bar{y})\left(y^{*}\right):=\left\{x^{*} \in X^{*} \mid\left(x^{*},-y^{*}\right) \in \widehat{N}((\bar{x}, \bar{y}) ; \operatorname{gph} F)\right\} .
$$

(ii) The normal Mordukhovich/Limiting Coderivative $D_{N}^{*} F(\bar{x}, \bar{y}): Y^{*} \rightrightarrows X^{*}$ of $F$ at $(\bar{x}, \bar{y})$ is defined via the normal cone to gph $F$ at $(\bar{x}, \bar{y})$ by

$$
\begin{aligned}
& D_{N}^{*} F(\bar{x}, \bar{y})\left(y^{*}\right):=\left\{x^{*} \in X^{*} \mid\left(x^{*},-y^{*}\right) \in N((\bar{x}, \bar{y}) ; \operatorname{gph} F)\right\} \\
&=\left\{x^{*} \in X^{*} \mid \exists\left(x_{k}, y_{k}\right) \stackrel{\operatorname{gph} F}{\longrightarrow}(\bar{x}, \bar{y}),\left(x_{k}^{*}, y_{k}^{*}\right) \stackrel{w^{*}}{\rightarrow}\left(x^{*}, y^{*}\right)\right. \\
&\text { with } \left.\left(x_{k}^{*},-y_{k}^{*}\right) \in \widehat{N}\left(\left(x_{k}, y_{k}\right) ; \operatorname{gph} F\right)\right\} .
\end{aligned}
$$

(iii) The mixed Mordukhovich/Limiting Coderivative $D_{M}^{*} F(\bar{x}, \bar{y}): Y^{*} \rightrightarrows X^{*}$ is defined by replacing the weak* convergence $y_{k}^{*} \stackrel{w^{*}}{\rightarrow} y^{*}$ in (2.10) with the norm convergence $y_{k}^{*} \stackrel{\|\cdot\|}{\rightarrow} y^{*}$, i.e.,

$$
\begin{array}{r}
D_{M}^{*} F(\bar{x}, \bar{y})\left(y^{*}\right):=\left\{x^{*} \in X^{*} \mid \quad \exists\left(x_{k}, y_{k}\right) \stackrel{\operatorname{gph} F}{\longrightarrow}(\bar{x}, \bar{y}), x_{k}^{*} \stackrel{w^{*}}{\rightarrow} x^{*}, y_{k}^{*} \stackrel{\|\cdot\|_{1}}{\longrightarrow} y^{*}\right. \\
\text { with } \left.\left(x_{k}^{*},-y_{k}^{*}\right) \in \widehat{N}\left(\left(x_{k}, y_{k}\right) ; \operatorname{gph} F\right)\right\} .
\end{array}
$$

Note that $\bar{y}=f(\bar{x})$ is always omitted in the coderivative notation if $F=f: X \rightarrow Y$ is single-valued. Obviously, for every $y^{*} \in Y^{*}$ we have

$$
D_{M}^{*} F(\bar{x}, \bar{y})\left(y^{*}\right) \subseteq D_{N}^{*} F(\bar{x}, \bar{y})\left(y^{*}\right),
$$

where the equality holds if $Y$ is finite-dimensional. If $f$ is strictly differentiable at $\bar{x}$ (which is automatic when it is $C^{1}$ around this point), then (2.12) becomes

$$
D_{N}^{*} f(\bar{x})\left(y^{*}\right)=D_{M}^{*} f(\bar{x})\left(y^{*}\right)=\nabla f(\bar{x})^{*} y^{*},
$$


where $\nabla f(\bar{x})$ is the Fréchet gradient of $f$ at $\bar{x}$.

A significant advantage of the mixed coderivative is the pointbased characterization of Lipschitz-like behavior to set-valued mappings. We denote the unit ball in $Y$ by $\mathbb{B}$.

Definition 2.5. (Lipschitz-like behavior). Let $F: X \rightrightarrows Y$ be a set-valued mapping between Asplund spaces. It is said to be LIPSCHITZ-LIKE (known also as $F$ enjoys Aubin's "pseudo-Lipschitzian" property) around a point $(\bar{x}, \bar{y}) \in \operatorname{gph} F$ if there are neighborhoods $U$ of $\bar{x}$ and $V$ of $\bar{y}$ and a nonnegative number $\ell \geq 0$ such that

$$
\forall x, u \in U: F(x) \cap V \subseteq F(u)+\ell\|x-u\| \mathbb{B} .
$$

This robust Lipschitzian property of $F$ is known to be equivalent to both metric regularity and linear openness properties of the inverse multifunction. Recall that $F$ has the local covering property around $(\bar{x}, \bar{y})$ with modulus $\kappa \geq 0$ if there are neighborhoods $U$ of $\bar{x}$ and $V$ of $\bar{y}$ such that

$$
F(x) \cap V+\kappa r \mathbb{B} \subseteq F(x+r \mathbb{B}) \text { whenever } x+r \mathbb{B} \subseteq U \text { as } r>0 .
$$

In infinite-dimensional spaces, the validity of calculus and characterizations for these generalized differentiation requires certain additional "sequential normal compactness" properties of sets and mappings, which are automatic in finite dimensions, while being a crucial ingredient of variational analysis in infinite dimensions.

Definition 2.6. (SNC and PSNC properties). Given a set $\Omega \subseteq X \times Y$ in the product of Asplund spaces; in particular, $\Omega=\operatorname{gph} F$, where $F: X \rightrightarrows Y$ is a set-valued mapping. Assume that $\Omega$ is locally closed around $(\bar{x}, \bar{y}) \in \Omega$.

(i) $\Omega$ is Sequentially NORMALly COMPACt (abrev. SNC) at $\bar{v}=(\bar{x}, \bar{y})$ if for any sequences $\left\{v_{k}, x_{k}^{*}, y_{k}^{*}\right\}$ satisfying

$$
v_{k} \stackrel{\Omega}{\rightarrow} \bar{v},\left(x_{k}^{*}, y_{k}^{*}\right) \in \widehat{N}\left(v_{k} ; \Omega\right)(k \in \mathbb{N}),
$$

one has

$$
\left(x_{k}^{*}, y_{k}^{*}\right) \stackrel{w^{*}}{\rightarrow} \mathbf{0} \Longrightarrow\left(x_{k}^{*}, y_{k}^{*}\right) \stackrel{\|\cdot\|}{\longrightarrow} \mathbf{0}
$$

The product structure of the space in question plays no role in this property (we can put $Y=\{\mathbf{0}\}$ without loss of generality) in contrast to its following partial modifications.

(ii) $\Omega$ is PARTIALLY SEQUENTIALLY NORMALLY COMPACT (abrev. PSNC) with respect to $X$ at $\bar{v}$ if for any sequences $\left(v_{k}, x_{k}^{*}, y_{k}^{*}\right)$ satisfying (2.13) one has

$$
\left(x_{k}^{*} \stackrel{w^{*}}{\rightarrow} \mathbf{0} \wedge y_{k}^{*} \stackrel{\|\cdot\|}{\longrightarrow} \mathbf{0}\right) \Longrightarrow x_{k}^{*} \stackrel{\|\cdot\|}{\longrightarrow} \mathbf{0} .
$$

(iii) $F$ is $\mathrm{SNC}$ at $(\bar{x}, \bar{y})$ if $\operatorname{gph} F$ is $\mathrm{SNC}$ at $(\bar{x}, \bar{y})$.

(iv) $F$ is PSNC at $(\bar{x}, \bar{z})$ if gph $F$ is PSNC at $(\bar{x}, \bar{y})$ with respect to $X$.

The next lemma provides a sufficient condition for the SNC property for sets established in [20, Theorem 1.21].

Lemma 2.5. (sufficient conditions for SNC conditions). A set $\Omega$ in an Asplund space $X$ is $S N C$ at $\bar{x} \in \Omega$ only if

$$
\operatorname{codim} \overline{\operatorname{aff}}(\Omega \cap U)<\infty
$$


for any neighborhood $U$ of $\bar{x}$, where $\overline{\operatorname{aff}}(A)$ is the closed affine hull of $A$ and codim $\overline{\operatorname{aff}}(A)$ is defined as the dimension of the quotient space $X /(\overline{\mathrm{aff}}-x)$. In particular, a singleton in $X$ is SNC if and only if $X$ is finite-dimensional. Moreover, when $\Omega$ is convex and $\operatorname{ri}(\Omega) \neq \emptyset$, the sequential normal compactness of $\Omega$ at every $\bar{x} \in \Omega$ is equivalent to the finite codimension condition $\operatorname{codim} \overline{\operatorname{aff}}(\Omega \cap U)<\infty$.

Assume in addition that the space $Y$ is equipped with a domination set $\Theta$ of $Y$. Then, the epigraph of $F$ with respect to $\Theta$ is defined by

$$
\text { epi } F:=\{(x, y) \in X \times Y \mid y \in F(x)+\Theta\} \text {; }
$$

we omit $\Theta$ in the epigraph notation for simplicity. We call the set-valued mapping $\mathscr{E}_{F}: X \rightrightarrows Y$ defined by

$$
\mathscr{E}_{F}(x):=F(x)+\Theta
$$

the epigraphical multifunction with $F$ (and $\Theta$ ) due to the fact that $\operatorname{gph} \mathscr{E}_{F}=\mathrm{epi} F$. Adopting coderivatives of set-valued mappings to epigraphical multifunctions, we define subdifferential constructions for $F$.

Definition 2.7. (subdifferentials of set-valued mappings). Let $F: X \rightrightarrows Y$ be a set-valued mapping acting between two Asplund spaces and $\Theta$ be a domination set of $Y$. Assume that epi $F$ is locally closed at $(\bar{x}, \bar{y}) \in \operatorname{epi} F$.

(i) The Regular subdifferential $\widehat{\partial} F(\bar{x}, \bar{y}): Y^{*} \rightrightarrows X^{*}$ of $F$ at $(\bar{x}, \bar{y})$ is defined by

$$
\widehat{\partial} F(\bar{x}, \bar{y})\left(y^{*}\right):=\widehat{D}^{*} \mathscr{E}_{F}(\bar{x}, \bar{y})\left(y^{*}\right) .
$$

(ii) The BASIC Subdifferential $\partial F(\bar{x}, \bar{y}): Y^{*} \rightrightarrows X^{*}$ of $F$ at $(\bar{x}, \bar{y})$ is defined by

$$
\partial F(\bar{x}, \bar{y})\left(y^{*}\right):=D_{N}^{*} \mathscr{E}_{F}(\bar{x}, \bar{y})\left(y^{*}\right) .
$$

(iii) The Singular SubdifFEREnTial $\partial^{\infty} F(\bar{x}, \bar{y})$ of $F$ at $(\bar{x}, \bar{y})$ is defined by

$$
\partial^{\infty} F(\bar{x}, \bar{y}):=D_{M}^{*} \mathscr{E}_{F}(\bar{x}, \bar{y})(\mathbf{0}) .
$$

As usual, we drop $\bar{y}=f(\bar{x})$ in the subdifferential notations (2.15) and (2.16) when $F=f: X \rightarrow Y$ is single-valued.

When $F=\varphi: X \rightarrow \mathbb{R} \cup\{ \pm \infty\}$ is an extended-real-valued functional finite at $\bar{x}$ with the standard ordering cone $\Theta=\mathbb{R}_{+}$on $\mathbb{R}$, the epigraphical multifunction (2.14) reduces to the known mapping in scalar optimization $\mathscr{E} \varphi(x)=\{\alpha \in \mathbb{R} \mid \alpha \geq \varphi(x)\}$ and the subdifferential (2.15) with $y^{*}=1$ and $\bar{y}=\varphi(\bar{x})$, and the singular subdifferential (2.16) become the well-known prototypes

$$
\begin{aligned}
\partial \varphi(\bar{x}) & :=\left\{x^{*} \in X^{*} \mid\left(x^{*},-1\right) \in N((\bar{x}, \varphi(\bar{x})) ; \text { epi } \varphi)\right\} \text { and } \\
\partial^{\infty} \varphi(\bar{x}) & :=\left\{x^{*} \in X^{*} \mid\left(x^{*}, 0\right) \in N((\bar{x}, \varphi(\bar{x})) ; \text { epi } \varphi)\right\} .
\end{aligned}
$$

Definition 2.8. (sequential normal epi-compactness of functionals). Let $\varphi: X \rightarrow \mathbb{R} \cup\{ \pm \infty\}$ be finite at $\bar{x}$. We say that $\varphi$ is SEQUENTIAL NORMAL EPI-COMPACT (abrev. SNEC) at $\bar{x}$ if its epigraph is SNC at $(\bar{x}, \varphi(\bar{x}))$.

To close this subsection, we recall the pointbased characterizations of Lipschitz-like property in [20, Theorem 4.10]. 
Lemma 2.6. (pointbased characterizations of Lipschitz-like property). Let $F: X \rightrightarrows Y$ be a setvalued mapping between Asplund spaces and assume that $\operatorname{gph} F$ is locally closed around $(\bar{x}, \bar{y}) \in \operatorname{gph} F$. Then, the following properties are equivalent:

(a) $F$ is Lipschitz-like around $(\bar{x}, \bar{y})$.

(b) $F$ is PSNC at $(\bar{x}, \bar{y})$ and $D_{M}^{*} F(\bar{x}, \bar{y})(\mathbf{0})=\{\mathbf{0}\}$.

As a consequence, a functional $\varphi: X \rightarrow \mathbb{R} \cup\{ \pm \infty\}$ is locally Lipschitz at $\bar{x}$ if and only if $\partial^{\infty} \varphi(\bar{x})=\{\mathbf{0}\}$.

\section{SUBDIFFERENTIALS OF SCALARIZATION FUNCTIONALS}

In this section, we establish formulas for both basic and singular subdifferentials of the scalarization functional $\varphi_{A, \mathbf{k}}$ defined in Definition 2.2. In addition, we study conditions ensuring the SNEC property and the Lipschitz behavior for $\varphi_{A, \mathbf{k}}$. In comparison with Lemma 2.3, we do not assume that the set $A$ is a cone and that the set $A$ is convex and/or solid. When $A$ is not convex, the scalarization functional $\varphi_{A, \mathbf{k}}$ is not convex. When $A$ is nonsolid, it is not Lipschitz. Therefore, we use the limiting generalized differentiation to compute both regular and limiting subdifferentials of the scalarization functional. (see Corollary 3.1 for the basic and singular subdifferentials)

Theorem 3.1. (subdifferentials of scalarization functionals). Let $Y$ be an Asplund space, A be a proper and closed set in $Y$, and $\mathbf{k} \in \operatorname{dir}(A)$ be a scalarization direction of $A$. Consider the scalarization functional $\varphi_{A, \mathbf{k}}$ introduced in Definition 2.2 and $y \in \operatorname{dom} \varphi_{A, \mathbf{k}}$. Then, the regular and limiting subdifferentials of $\varphi_{A, \mathbf{k}}$ at $(\bar{y}, \bar{t}) \in \operatorname{epi} \varphi_{A, \mathbf{k}}$ are given by (for $\lambda \in \mathbb{R}$ )

$$
\partial_{\bullet} \varphi_{A, \mathbf{k}}(\bar{y})(\lambda)=H_{\lambda}(\mathbf{k}) \cap\left(-N_{\bullet}(\bar{t} \mathbf{k}-\bar{y} ; A)\right),
$$

where $H_{\lambda}(\mathbf{k}):=\left\{y^{*} \in Y^{*} \mid y^{*}(\mathbf{k})=\lambda\right\}$, and $\bullet$ stands for both regular and limiting constructions.

Proof. Define a set-valued mapping $F: \mathbb{R} \rightrightarrows Y$ by $F(t)=t \mathbf{k}-A$. We claim that $\operatorname{gph} F^{-1}=\operatorname{epi} \varphi_{A, \mathbf{k}}$. First, let us show that epi $\varphi_{A, \mathbf{k}} \subseteq \operatorname{gph} F^{-1}$. For any $(-u, t) \in \operatorname{epi} \varphi_{A, \mathbf{k}}$, we have $\varphi_{A, \mathbf{k}}(t \mathbf{k}-u-t \mathbf{k}) \leq t$. By Lemma 2.1, we have $\varphi_{A, \mathbf{k}}(-u-t \mathbf{k})+t \leq t$ and thus $\varphi_{A, \mathbf{k}}(-u-t \mathbf{k}) \leq 0$. The latter implies $-u-t \mathbf{k} \in-A$. Set $y=u+t \mathbf{k} \in A$. We have $(-u, t)=(t \mathbf{k}-y, t) \in \operatorname{gph} F^{-1}$. To show the reverse inclusion, we fix an arbitrary pair $(t, t \mathbf{k}-y) \in \operatorname{gph} F$ implying that $y \in A$ and $(t \mathbf{k}-y, t) \in \operatorname{gph} F$. By Lemma 2.1, we have $\varphi_{A, \mathbf{k}}(-y) \leq 0$ and $\varphi_{A, \mathbf{k}}(t \mathbf{k}-y)=t+\varphi_{A, \mathbf{k}}(-y) \leq t$ clearly verifying that $(t \mathbf{k}-y, t) \in \operatorname{epi} \varphi_{A, \mathbf{k}}$. Since $(t \mathbf{k}-y, t)$ was arbitrary in $\operatorname{gph} F^{-1}$, we have gph $F^{-1} \subseteq$ epi $\varphi_{A, \mathbf{k}}$.

Next, we introduce a constant set-valued mapping $\mathscr{A}: \mathbb{R} \rightrightarrows Y$ with $\mathscr{A}(t) \equiv-A$ and a strictly differentiable function $\kappa: \mathbb{R} \rightarrow Y$ with $\kappa(t)=t \mathbf{k}$ to describe the set-valued mapping $F$ as a sum of two mappings $F=\kappa+\mathscr{A}$. Fix an arbitrary pair $(\bar{t}, \bar{y}) \in \operatorname{gph} F$, i.e., $(\bar{y}, \bar{t}) \in \operatorname{epi} \varphi_{A, \mathbf{k}}$. We apply the coderivative sum rule with equality from [20, Theorem 1.62] to $\kappa$ and $\mathscr{A}$. For every $y^{*} \in Y^{*}$, we have

$$
D^{*} F(\bar{t}, \bar{y})\left(-y^{*}\right)=-y^{*}(\mathbf{k})+D^{*} \mathscr{A}(\bar{t}, \bar{y}-\bar{t} \mathbf{k})\left(-y^{*}\right)
$$

where $D^{*} F(\bar{t}, \bar{y})$ stands for both the regular coderivative $\widehat{D}^{*} F(\bar{t}, \bar{y})$ and the limiting coderivative $D_{N}^{*} F(\bar{t}, \bar{y})$ defined in Definition 2.4.

Since gph $\mathscr{A}=\mathbb{R} \times(-A)$, [20, Proposition 1.2] stating that the normal cone to Cartesian sets is the product of the normal cones to component sets yields

$$
N_{\bullet}((\bar{t}, \bar{y}-\bar{t} \mathbf{k}) ; \operatorname{gph} \mathscr{A})=N_{\bullet}(\bar{t} ; \mathbb{R}) \times N_{\bullet}(\bar{y}-\bar{t} \mathbf{k} ;-A)=\{\mathbf{0}\} \times N_{\bullet}(\bar{y}-\bar{t} \mathbf{k} ;-A),
$$


where $N_{\bullet}((\bar{t}, \bar{y}-\bar{t} \mathbf{k})$; gph $\mathscr{A})$ stands for both the regular normal cone $\widehat{N}((\bar{t}, \bar{y}-\bar{t} \mathbf{k}) ; \operatorname{gph} \mathscr{A})$ and limiting normal cone $N((\bar{t}, \bar{y}-\bar{t} \mathbf{k}) ;$ gph $\mathscr{A})$ defined in Definition 2.3. Taking into account Definition 2.4 for coderivatives, we have

$$
y^{*} \in N_{\bullet}(\bar{y}-\bar{t} \mathbf{k} ;-A) \text { and } D^{*} \mathscr{A}(\bar{y}-\bar{t} \mathbf{k} ;-A)\left(-y^{*}\right)=\{\mathbf{0}\} .
$$

We now get from (3.2) that $y^{*} \in N_{\bullet}(\bar{y}-\bar{t} \mathbf{k} ;-A)=-N_{\bullet}(\bar{t} \mathbf{k}-\bar{y} ; A)$ and $D^{*} F(\bar{t}, \bar{y})\left(-y^{*}\right)=-y^{*}(\mathbf{k})$. Further manipulate the latter equality, we have

$$
\begin{aligned}
&\left(-y^{*}(\mathbf{k}), y^{*}\right) \in N_{\bullet}((\bar{t}, \bar{y}) ; \operatorname{gph} F) \Leftrightarrow\left(y^{*},-y^{*}(\mathbf{k})\right) \in N_{\bullet}\left((\bar{y}, \bar{t}) ; \operatorname{gph} F^{-1}\right) \\
& \Longleftrightarrow \quad\left(y^{*},-y^{*}(\mathbf{k})\right) \in N_{\bullet}\left((\bar{y}, \bar{t}) ; \operatorname{epi} \varphi_{A, \mathbf{k}}\right) \Longleftrightarrow y^{*} \in D^{*} \mathscr{E}_{\varphi_{A, \mathbf{k}}}(\bar{y}, \bar{t})\left(y^{*}(\mathbf{k})\right) .
\end{aligned}
$$

Taking into account Definition 2.7, we get that

$$
\partial_{\bullet} \varphi_{A, \mathbf{k}}(\bar{y}, \bar{t})(\lambda)=D^{*} \mathscr{E}_{\varphi_{A, \mathbf{k}}}(\bar{y}, \bar{t})(\lambda)=H_{\lambda}(\mathbf{k}) \cap\left(-N_{\bullet}(\bar{t} \mathbf{k}-\bar{y} ; A)\right),
$$

where $H_{\lambda}(\mathbf{k})=\left\{y^{*} \in Y^{*} \mid y^{*}(\mathbf{k})=\lambda\right\}$. The proof is complete.

Corollary 3.1. (subdifferentials of scalarization functionals). Let $Y, A, \mathbf{k}, \varphi_{A, \mathbf{k}},(\bar{y}, \bar{t})$ as in Theorem 3.1. Then:

(i) The basic subdifferential of $\varphi_{A, \mathbf{k}}$ at $\bar{y}$ is

$$
\partial \varphi_{A, \mathbf{k}}(\bar{y})=H_{1}(\mathbf{k}) \cap\left(-N\left(\varphi_{A, \mathbf{k}}(\bar{y}) \mathbf{k}-\bar{y} ; A\right)\right),
$$

where $H_{1}(\mathbf{k}):=\left\{y^{*} \in Y^{*} \mid y^{*}(\mathbf{k})=1\right\}$.

(ii) The singular subdifferential of $\varphi_{A, \mathbf{k}}$ at $\bar{y}$ is

$$
\partial^{\infty} \varphi_{A, \mathbf{k}}(\bar{y})=H_{0}(\mathbf{k}) \cap\left(-N\left(\varphi_{A, \mathbf{k}}(\bar{y}) \mathbf{k}-\bar{y} ; A\right)\right),
$$

where $H_{0}(\mathbf{k}):=\left\{y^{*} \in Y^{*} \mid y^{*}(\mathbf{k})=0\right\}$.

\section{Remark 3.1. (some special cases).}

(a) Corollary 3.1 improves the results in [5] in which the formulas were established via bd $(A)$ instead of $A$. Theorem 3.1 provides subdifferentials of the scalarization functionals at pairs in the epigraph which are essential for us to study the equivalence between the SNC property of the set $A$ and the SNEC property of the scalarization functional $\varphi_{A, \mathbf{k}}$. As mentioned before, since we do not assume that the set $A$ is solid, we need to compute the singular subdifferential $\partial^{\infty} \varphi_{C, \mathbf{k}}$ of $\varphi_{A, \mathbf{k}}$ in order to verify the fulfillment of the so-called qualification condition of calculus rules for generalized differentiation.

(b) When $A=C$ is convex, both regular and limiting normal cone to $C$ reduces to the normal cone of convex analysis. Therefore, the basic subdifferential (3.4) of $\varphi_{C, \mathbf{k}}$ reduces to the known formulas in Lemmas 2.2 and 2.3 (with less restrictive assumptions imposed on $C$ ). Precisely, we have

$$
\begin{aligned}
\partial \varphi_{C, \mathbf{k}}(\bar{y}) & =H_{1}(\mathbf{k}) \cap\left(-N\left(\varphi_{C, \mathbf{k}}(\bar{y}) \mathbf{k}-\bar{y} ; C\right)\right) \\
& =H_{1}(\mathbf{k}) \cap\left\{y^{*} \in Y^{*} \mid \forall y \in C: y^{*}(y+\bar{y})-\varphi_{C, \mathbf{k}}(\bar{y}) \geq 0\right\} \\
& =H_{1}(\mathbf{k}) \cap\left\{y^{*} \in Y^{*} \mid y^{*}(\bar{y})=\varphi_{C, \mathbf{k}}(\bar{y})\right\},
\end{aligned}
$$

where the second equality holds for any convex set $C$ and the last equality is valid for any convex cone $C$. 
(c) When $A=C$ is a nontrivial, convex and solid cone and $\mathbf{k} \in \operatorname{int} \Theta$; in this setting, $\operatorname{dom} \varphi_{C, \mathbf{k}}=Y$ and the singular subdifferential $\partial^{\infty} \varphi_{C, \mathbf{k}}$ vanishes everywhere, i.e.,

$$
\partial^{\infty} \varphi_{C, \mathbf{k}}(\bar{y})=H_{0}(\mathbf{k}) \cap\left(-N\left(\varphi_{C, \mathbf{k}}(\bar{y}) \mathbf{k}-\bar{y} ; C\right)\right)=\{\mathbf{0}\} .
$$

As a consequence of Theorem 3.1, subdifferentials of scalarization functionals shifted along the scalarization direction.

Corollary 3.2. (subdifferentials along scalarization directions). Let $Y, A, \mathbf{k}, \varphi_{A, \mathbf{k}},(\bar{y}, \bar{t})$ as in Theorem 3.1. Then, for any $\bar{y} \in \operatorname{dom} \varphi_{A, \mathbf{k}}=\mathbb{R} \mathbf{k}-A$ and for any $\bar{t} \in \mathbb{R}$, we have $\partial \varphi_{A, \mathbf{k}}(\bar{y}+\bar{t} \mathbf{k})=\partial \varphi_{A, \mathbf{k}}(\bar{y})$ and $\partial^{\infty} \varphi_{A, \mathbf{k}}(\bar{y}+\bar{t} \mathbf{k})=\partial^{\infty} \varphi_{A, \mathbf{k}}(\bar{y})$.

Proof. The proof is straightforward due to Theorem 3.1 and Lemma 2.1(b) since

$$
\begin{aligned}
\partial \varphi_{A, \mathbf{k}}(\bar{y}+\bar{t} \mathbf{k})(\lambda) & =H_{\lambda}(\mathbf{k}) \cap\left(-N\left(\varphi_{A, \mathbf{k}}(\bar{y}+\bar{t} \mathbf{k}) \mathbf{k}-\bar{y}-\bar{t} \mathbf{k} ; A\right)\right) \\
& =H_{\lambda}(\mathbf{k}) \cap\left(-N\left(\varphi_{A, \mathbf{k}}(\bar{y}) \mathbf{k}+\bar{t} \mathbf{k}-\bar{y}-\bar{t} \mathbf{k} ; A\right)\right) \\
& =H_{\lambda}(\mathbf{k}) \cap\left(-N\left(\varphi_{A, \mathbf{k}}(\bar{y}) \mathbf{k}-\bar{y} ; A\right)\right)=\partial \varphi_{A, \mathbf{k}}(\bar{y})(\lambda),
\end{aligned}
$$

where $\lambda=1$ (resp. $\lambda=0$ ) stands for the basic (resp. singular) subdifferential and the second equality holds due to Lemma 2.1(b).

Next, we study Lipschitz behavior and SNEC property of the scalarization functional $\varphi_{A, \mathbf{k}}$.

Theorem 3.2. (SNEC property of scalarization functionals). Let $Y, A, \mathbf{k}, \varphi_{A, \mathbf{k}},(\bar{y}, \bar{t})$ as in Theorem 3.1. The scalarization functional $\varphi_{A, \mathbf{k}}$ defined in Definition 2.2 is $S N E C$ at $\bar{y} \in \operatorname{dom} \varphi_{A, \mathbf{k}}$ if and only if $A$ is SNC at $\varphi_{A, k}(\bar{y}) \mathbf{k}-\bar{y}$.

Proof. By Definitions 2.6 and 2.8 for SNC and SNEC properties, $\varphi_{A, \mathbf{k}}$ is SNEC at $\bar{y} \in \operatorname{dom} \varphi_{A, \mathbf{k}}$ if and only if epi $\varphi_{A, \mathbf{k}}$ is SNC at $\left(\bar{y}, \varphi_{A, \mathbf{k}}(\bar{y})\right)$. To justify the latter, fix an arbitrary sequence $\left(y_{k}^{*},-\lambda_{k}\right) \in$ $\widehat{N}\left(\left(y_{k}, \alpha_{k}\right)\right.$; epi $\left.\varphi_{A, \mathbf{k}}\right)$ such that $\left(y_{k}, \alpha_{k}\right) \rightarrow\left(\bar{y}, \varphi_{A, \mathbf{k}}(\bar{y})\right), x_{k}^{*} \stackrel{w^{*}}{\rightarrow} 0$, and $\lambda_{k} \rightarrow 0$ as $k \rightarrow \infty$. We get from $\left(y_{k}^{*},-\lambda_{k}\right) \in \widehat{N}\left(\left(y_{k}, \alpha_{k}\right)\right.$; epi $\left.\varphi_{A, \mathbf{k}}\right)$ that $y_{k}^{*} \in \widehat{\partial} \varphi_{A, \mathbf{k}}\left(y_{k}, \alpha_{k}\right)\left(\lambda_{k}\right)$. Taking into account the description of the regular subdifferential in Theorem 3.1, we have

$$
\lambda_{k}=y_{k}^{*}(\mathbf{k}) \text { and }-y_{k}^{*} \in \widehat{N}\left(\alpha_{k} \mathbf{k}-y_{k} ; A\right) .
$$

By Definition 2.6, $A$ is SNC at $\varphi_{A, \mathbf{k}}(\bar{y}) \mathbf{k}-\bar{y}$. The proof is complete.

Theorem 3.3. (characteristics of Lipschitz behavior of scalarization functionals). Let $Y, A, \mathbf{k}, \varphi_{A, \mathbf{k}}$, $(\bar{y}, \bar{t})$ as in Theorem 3.1. The scalarization functional $\varphi_{A, \mathbf{k}}$ defined in Definition 2.2 is locally Lipschitz continuous at $\bar{y} \in \operatorname{dom} \varphi_{A, \mathbf{k}}$ if and only if $A$ is $S N C$ at $\bar{v}:=\varphi_{A, \mathbf{k}}(\bar{y}) \mathbf{k}-\bar{y}$ and

$$
H_{0}(\mathbf{k}) \cap(-N(\bar{v} ; A))=\{\mathbf{0}\}
$$

where $H_{0}(\mathbf{k})=\left\{y^{*} \in Y^{*} \mid y^{*}(\mathbf{k})=0\right\}$.

Proof. By Theorem 3.1, we have

$$
\partial^{\infty} \varphi_{A, \mathbf{k}}(\bar{y})=H_{0}(\mathbf{k}) \cap(-N(\bar{v} ; A)) .
$$

By Corollary 3.2, $\varphi_{A, \mathbf{k}}$ is SNEC at $\bar{y}$. Therefore, Theorem 4.10 in [20], which tells us that an extendedreal-valued function is locally Lipschitz continuous at a point $\bar{y}$ in its domain if and only if its singular sudifferential is trivial and it is SNEC at that point, completes the proof. 
This Lipschitz result is different from [24, Theorem 3.9] stating that $\varphi_{A, \mathbf{k}}$ is finite and Lipschitz on a neighborhood of $\bar{y}$ if and only if $A$ is epi-Lipschitz at $\bar{v}:=\bar{y}-\varphi_{A, \mathbf{k}}(\bar{y}) \mathbf{k}$ in the direction $-\mathbf{k}$. Assume in addition that $A$ is a convex set, then the epi-Lipschitzian property of $A$ is equivalent to int $A \neq \emptyset$ and this implies the SNC property.

Let us illustrate how to use Theorem 3.3 to determine whether the scalarization functional $\varphi_{A, \mathbf{k}}$ is locally Lipschitz or not. Consider $Y=\mathbb{R}^{3}, A=\mathbb{R}_{+} \times \mathbb{R}_{+} \times\{0\}$ and $\mathbf{k}=(1,1,0)$. Obviously, dom $\varphi_{A, \mathbf{k}}=$ $\mathbb{R}^{2} \times\{0\}$, the SNC property is automatic in the finite dimensional space $\mathbb{R}^{3}$, and $H_{0}(\mathbf{k})=\{(a, b, c) \in$ $\left.\mathscr{R}^{3} \mid a+b=0\right\}$. To check the validity of condition (3.6), we consider four cases:

1. When $\bar{v}=(0,0,0)$, we have $N(\bar{v} ; A)=\mathbb{R}_{-} \times \mathbb{R}_{-} \times \mathbb{R}$.

2. When $\bar{v}=(a, 0,0)$ with $a>0$, we have $N(\bar{v} ; A)=\{0\} \times \mathbb{R}_{-} \times \mathbb{R}$.

3. When $\bar{v}=(0, b, 0)$ with $b>0$, we have $N(\bar{v} ; A)=\mathbb{R}_{-} \times\{0\} \times \mathbb{R}$.

4. When $\bar{v}=(a, b, 0)$ with $a>0$ and $b>0$, we have $N(\bar{v} ; A)=\{0\} \times\{0\} \times \mathbb{R}$.

Since condition (3.6) does not hold for each cases, the scalarization functional is not Lipschitz on $\operatorname{dom} \varphi_{A, \mathbf{k}}=\mathbb{R}^{2} \times\{0\}$.

Corollary 3.3. (Lipschitz continuity of scalarization functionals). Let $Y$ be an Asplund space, $C$ be a nontrivial, closed, convex and solid cone in $Y$, and $\mathbf{k} \in \operatorname{int}(C)$ be a scalarization direction of $C$. The, then scalarization functional $\varphi_{C, \mathbf{k}}$ defined in Definition 2.2 is locally Lipschitz.

Proof. By Lemma 2.1 (a) and $\mathbf{k} \in \operatorname{int} C$, we have $\operatorname{dom} \varphi_{A, \varphi}=\mathbb{R} \mathbf{k}-C=Y$. By Proposition 1.25 and Theorem 1.26 in [20], the solidness and convexity of $C$ implies the SNC property of $C$. By Theorem 3.2, $\varphi_{C, \mathbf{k}}$ is SNEC at every point $\bar{y} \in Y$.

Next we will show that $\partial^{\infty} \varphi_{C, \mathbf{k}}(\bar{y})=\{\mathbf{0}\}$. By Corollary 3.1(ii), it is sufficient to show that

$$
H_{0}(\mathbf{k}) \cap\left(-N\left(\varphi_{C, \mathbf{k}}(\bar{y}) \mathbf{k}-\bar{y} ; C\right)\right)=\{\mathbf{0}\} .
$$

Since $C$ is a convex cone, the point $\bar{v}:=\varphi_{C, \mathbf{k}}(\bar{y}) \mathbf{k}-\bar{y}$ is in $C$. We get from $\mathbf{k} \in \operatorname{int} C$ the existence of $r>0$ such that $\bar{v}+\mathbf{k}+\mathbb{B}_{r}(\mathbf{0}) \subseteq C$, where $\mathbb{B}_{r}(\mathbf{0})$ is the ball centered at the origin with radius $r$ in $Y$. For any $y^{*} \in H_{0}(\mathbf{k}) \cap(-N(\bar{v} ; C))$, we have $y^{*}(\mathbf{k})=0$ and $\left.-y^{*} \in N(\bar{v} ; C)\right)$, i.e., for every $y \in C$ we have $y^{*}(y-\bar{v}) \geq 0$. Then, for all $u \in \mathbb{B}_{r}(\mathbf{0})$, we have $\bar{y}=\bar{v}+\mathbf{k}+u \in C$ and thus $y^{*}(u) \geq 0$ clearly verifying that $y^{*}=\mathbf{0}$. By Theorem 3.3, $\varphi_{C, \mathbf{k}}$ is locally Lipschitz at every point $\bar{y} \in Y$.

\section{APPLICATIONS TO OPTIMIZATION PROBLEMS WITH SET-VALUED OBJECTIVES}

This section is devoted to establishing necessary conditions to the class of vector optimization problems

$$
\Theta-\text { Minimize } F(x) \text { subject to } x \in \Omega \text {, }
$$

where $F: X \rightrightarrows Y$ is a set-valued mapping acting between two Asplund spaces, $\Omega$ is a nonempty subset of $X, \Theta$ is a domination set in $Y$ with $\operatorname{dir}(\Theta) \neq \emptyset$, and the " $\Theta$-minimality" is understood in the following sense.

Definition 4.1. ( $\Theta$-minimality). Consider problem (4.1). Let $\bar{x} \in \Omega$ and $(\bar{x}, \bar{y}) \in \operatorname{gph} F$. We say that the pair $(\bar{x}, \bar{y}) \in \operatorname{gph} F$ is a $\Theta$-minimal solution of problem (4.1) if

$$
F(\Omega) \cap(\bar{y}-\Theta)=\{\bar{y}\} .
$$


When $\Omega=X$, problem (4.1) becomes an unconstrained optimization problem with set-valued objectives

$$
\Theta-\text { Minimize } F(x),
$$

and a $\Theta$-minimal solution of problem (4.1) with $\Omega=X$ is called a $\Theta$-minimal solution of $F$.

It is worth mentioning that we use the vector approach to define the $\Theta$-minimality concept for setvalued mappings $F$ and thus we have to fix an element $\bar{y} \in F(\bar{x})$. This solution concept is very useful in establishing necessary conditions in terms of generalized differentiation. It is of increasing interest to study solution concepts of set-valued mappings defined via set inclusions proposed by Kuroiwa in [19] which is out of the scope of this paper and would be studied in the future. However, it is possible to derive necessary optimality conditions for solutions based on the concept of the set approach by Kuroiwa [19] using the results for solutions based on the vector approach and the relationships between both approaches (compare Eichfelder and Pilecka [11, 12], Köbis, Tammer and Yao [18]). This means that it is possible to use our necessary conditions for solutions defined by the vector approach for deriving corresponding results for solutions based on set approach.

It is important to emphasize that the domination set $\Theta$ is a far-going extension of the ordering cone in vector optimization. It allows us to capture the so-called $\varphi$-minimal solution, where $\varphi: Y \rightarrow \mathbb{R}$ is a utility function, in the sense that $\varphi(y)>\varphi(\bar{y})$ for all $y \in F(\Omega) \backslash\{\bar{y}\}$. In this setting, we have $\Theta:=-\{y \in Y \mid \varphi(y) \leq \varphi(\bar{y})\}-\bar{y}$.

Scalarization is an important stool in vector optimization. It is very effective in deriving necessary conditions for Pareto minimal solutions to vector optimization problems. Let us illustrate this approach in set-valued optimization.

It is very easy to check that for every scalarization direction $\mathbf{k} \in \operatorname{dir}(\Theta)$, if $(\bar{x}, \bar{y})$ is a $\Theta$-minimal solution of problem (4.2), then $(\bar{x}, \mathbf{0})$ is a scalarized minimal solution of the scalar set-valued mapping $\varphi_{\Theta-\bar{y}, \mathbf{k}} \circ F$. Employing the generalized Fermat rule, we get

$$
0 \in \partial\left(\varphi_{\Theta-\bar{y}, \mathbf{k}} \circ F\right)(\bar{x}, 0)(1) .
$$

To further manipulate the right-hand side, we use a chain rule for coderivetives and thus we need to assume that the set-valued mapping $S: X \times \mathbb{R} \rightrightarrows Y$ defined by

$$
S(x, \alpha):=\mathscr{E}_{F}(x) \cap \varphi^{-1}(\alpha)=\mathscr{E}_{F}(x) \cap(\alpha \mathbf{k}+\bar{y}-\Theta) .
$$

is inner semicontinuous at $(\bar{x}, \bar{\alpha}, \bar{y})$ in the sense that for every sequence $\left(x_{n}, \alpha_{n}\right)$ with $\lim _{n \rightarrow \infty}\left(x_{n}, \alpha_{n}\right)=$ $(\bar{x}, \bar{\alpha})$, there exists a sequence $y_{n} \in S\left(x_{n}, \alpha_{n}\right)$ such that $\lim _{n \rightarrow \infty} y_{n}=\bar{y}$. It is obvious that it is additional condition; i.e., it is not automatic when $\operatorname{gph} F$ is locally closed around $(\bar{x}, \bar{y})$ and $\Theta$ is a closed domination set.

In this paper, we modify the way to scalarize a set-valued optimization problem by introducing a scalarization functional acting from the product space $X \times Y$ into the extended real-valued numbers; see Proposition 4.1 below. Define an extended-real-valued functional $s: X \times Y \rightarrow \overline{\mathbb{R}}$ by

$$
s(x, y):=\varphi_{(\Theta-\bar{y}), \mathbf{k}}(\bar{y}) .
$$

Doing this way, we will avoid the usage of the chain rule for coderivatives to manipulate the subdifferential of the generalized composition $\varphi_{\Theta-\bar{y}, \mathbf{k}} \circ F$. 
We have the following relations between $\Theta$-minimal solutions of a set-valued mapping $F$ (with respect to a domination set $\Theta$ ) and minima of some scalarized optimality problem.

Proposition 4.1. (characteristics of scalarized solutions). Consider problem (4.2) with $\operatorname{dir}(\Theta) \neq \emptyset$. Let $(\bar{x}, \bar{y}) \in \operatorname{gph} F$. Then, the following hold:

(i) If $(\bar{x}, \bar{y})$ is a $\Theta$-minimal solution of problem (4.2), then for any $\mathbf{k} \in \operatorname{dir}(\Theta)$, the pair $(\bar{x}, \bar{y})$ is a minimum of the scalar optimization problem

$$
\text { minimize } s(x, y)+\delta((x, y) ; \operatorname{gph} F)
$$

where $s$ is defined by (4.3) and $\delta(\cdot ; \operatorname{gph} F): X \times Y \rightarrow \mathbb{R} \cup\{+\infty\}$ is the indication function of $\operatorname{gph} F$.

(ii) Assume additionally that $\Theta$ is closed and pointed and that

$$
\forall(x, y) \in \operatorname{gph} F: \varphi_{\Theta-\bar{y}, \mathbf{k}}(y)=0 \Rightarrow \varphi_{\Theta-y, \mathbf{k}}(\bar{y})=0 .
$$

If $(\bar{x}, \bar{y})$ is a minimum of the scalar optimization problem (4.4) for some $\mathbf{k} \in \operatorname{dir}(\Theta)$, then it is a $\Theta$-minimal solution of problem (4.2).

Proof. (i) Assume that $(\bar{x}, \bar{y})$ is a $\Theta$-minimal solution of $F$, i.e.,

$$
F(X) \cap(\bar{y}-\Theta)=\{\bar{y}\} .
$$

By Lemma 2.1 (a), for every $y \in F(X)$ we have $\varphi_{\Theta-\bar{y}, \mathbf{k}}(y) \geq 0$. Therefore,

$$
\forall(x, y) \in \operatorname{gph} F: s(x, y)=\varphi_{\Theta-\bar{y}, \mathbf{k}}(y) \geq 0 .
$$

Since $\Theta$ is a closed domination set, we have $s(\bar{x}, \bar{y})=\varphi_{\Theta-\bar{y}, \mathbf{k}}(\bar{y})=0$. Then, the pair $(\bar{x}, \bar{y})$ is a minimum of the scalar optimization problem (4.4).

(ii) Assume now that $(\bar{x}, \bar{y})$ is a minimum of the scalar optimization problem (4.4), i.e.,

$$
\forall x \in \operatorname{dom} F, \forall y \in F(x): \varphi_{\Theta-\bar{y}, \mathbf{k}}(y) \geq 0 .
$$

Arguing by contradiction, assume that $(\bar{x}, \bar{y})$ is not a $\Theta$-minimal solution of problem (4.2). Then, we could find an element $x \in \operatorname{dom} F$ and an element $y \in F(x)$ such that $y \neq \bar{y}$ and $y \in \bar{y}-\Theta$. The latter implies that $\varphi_{\Theta-\bar{y}, \mathbf{k}}(y) \leq 0$ and thus $\varphi_{\Theta-\bar{y}, \mathbf{k}}(y)=0$ due to the minimality of $(\bar{x}, \bar{y})$. Condition (4.5) ensures that $\varphi_{\Theta-y, \mathbf{k}}(\bar{y})=0$. Since $\Theta$ is closed, Lemma 2.1 (a) ensures $\bar{y} \in y-\Theta$. Since $\Theta$ is pointed, we get from $y \in \bar{y}-\Theta$ and $\bar{y} \in y-\Theta$ that $\bar{y}=y$ contradicting to the choice of $y(y \neq \bar{y})$. This contradiction justifies the $\Theta$-minimality of $(\bar{x}, \bar{y})$ to problem (4.2).

In order to derive necessary conditions for $\Theta$-minimal solutions of set-valued mappings, we need [20, Lemma 3.1] and [20, Theorem 2.60].

Lemma 4.1. (a fuzzy intersection rule from the extremal principle). Let $X$ be an Asplund space. Let $\Omega_{1}, \Omega_{2} \subseteq X$ be arbitrary sets locally closed around $\bar{x} \in \Omega_{1} \cap \Omega_{2}$, and let $x^{*} \in \widehat{N}\left(\bar{x} ; \Omega_{1} \cap \Omega_{2}\right)$. Then for every $\varepsilon>0$, there are $\lambda \geq 0, x_{i} \in \Omega_{i} \cap(\bar{x}+\varepsilon \mathbb{B})$, and $x_{i}^{*} \in \widehat{N}\left(x_{i} ; \Omega_{i}\right)+\varepsilon \mathbb{B}^{*}$ for $i=1,2$ such that

$$
\lambda x^{*}=x_{1}^{*}+x_{2}^{*} \text { and } \max \left\{\lambda, x_{1}^{*}\right\}=1 .
$$


Lemma 4.2. (subdifferential variational principle). Let $X$ be an Asplund space and $\varphi: X \rightarrow \mathbb{R} \cup\{+\infty\}$ be a proper lower semicontinuous function being bounded from below. For every $\varepsilon>0, \lambda>0$, and $x_{0} \in X$ with $\varphi\left(x_{0}\right)<\inf _{X} \varphi+\varepsilon$, there are $\bar{x} \in X$ and $x^{*} \in \widehat{\partial} \varphi(\bar{x})$ such that $\left\|\bar{x}-x_{0}\right\|<\lambda, \varphi(\bar{x})<\inf _{X} \varphi+\varepsilon$, and $\left\|x^{*}\right\|<\varepsilon / \lambda$.

Theorem 4.1. (necessary conditions for $\Theta$-minimal solutions of set-valued mappings). Consider problem (4.2). Let $(\bar{x}, \bar{y})$ be a $\Theta$-minimal solution of $F, \mathbf{k} \in \operatorname{dir}(\Theta)$ be a scalarization direction of $\Theta$, $\varphi_{\Theta-\bar{y}, \mathbf{k}}$ be the scalarization functional defined in Definition 2.2, and $s: X \times Y \rightarrow \overline{\mathbb{R}}$ be the scalarization functional defined by (4.3). Assume that

(H1) (closedness condition) The domination set $\Theta$ is locally closed around $\mathbf{0} \in \Theta$ and $\operatorname{gph} F$ is locally closed around $(\bar{x}, \bar{y})$.

(H2) (PSNC condition) Either $\Theta$ is SNC at $\mathbf{0}$ or $F^{-1}$ is PSNC at $(\bar{y}, \bar{x})$.

(H3) (mixed qualification condition for $\{F, \Theta\}$ )

$$
\left[y^{*} \in D_{M}^{*} F^{-1}(\bar{y}, \bar{x})(\mathbf{0}) \cap(-N(\mathbf{0} ; \Theta)) \text { and } y^{*}(\mathbf{k})=0\right] \Rightarrow y^{*}=\mathbf{0} .
$$

Then, there is $y^{*} \in-N(\mathbf{0} ; \Theta)$ with $y^{*}(\mathbf{k})=1$ satisfying

$$
-y^{*} \in D_{M}^{*} F^{-1}(\bar{y}, \bar{x})(\mathbf{0})
$$

which clearly implies $\mathbf{0} \in D_{N}^{*}(\bar{x}, \bar{y})\left(y^{*}\right)$.

Proof. By Proposition 4.1, the pair $(\bar{x}, \bar{y})$ is a minimum of the scalar problem (4.4). Since the limiting generalized differential objects and SNC are local properties, we may assume without loss generality that $\Theta$ is a closed set. Since $\Theta$ is a closed set in $Y$, the scalarization functional $\varphi_{\Theta-\bar{y}, \mathbf{k}}$ is lower semi-continuous around $\bar{y}$, and so is the scalarization functional $s$ around $(\bar{x}, \bar{y})$. Fix a positive number sequence $\left\{\varepsilon_{k}\right\}$ with $\varepsilon_{k} \downarrow 0$. Employing the subdifferential variational principle in Lemma 4.2 to the $\varepsilon_{k}^{2}$-minimal solution of $g:=s+\delta(\cdot ; \operatorname{gph} F)$ with $\lambda=\varepsilon$, we could find a sequence $\left\{x_{k}, y_{k}, x_{k}^{*}, y_{k}^{*}\right\}$ satisfying

$$
\begin{aligned}
& \left(x_{k}, y_{k}\right) \in \operatorname{gph} F,\left\|\left(x_{k}, y_{k}\right)-(\bar{x}, \bar{y})\right\| \leq \varepsilon_{k}, \\
& \left(x_{k}^{*}, y_{k}^{*}\right) \in \widehat{\partial} g\left(x_{k}, y_{k}\right),\left\|\left(x_{k}^{*}, y_{k}^{*}\right)\right\| \leq \varepsilon_{k} .
\end{aligned}
$$

Since epi $g=\Omega_{1} \cap \Omega_{2}$, where $\Omega_{1}=\operatorname{epi} s=X \times \operatorname{epi} \varphi_{\Theta-\bar{y}, \mathbf{k}}$ and $\Omega_{2}=\operatorname{gph} F \times \mathbb{R}$, we get from $\left(x_{k}^{*}, y_{k}^{*}\right) \in$ $\widehat{\partial} g\left(x_{k}, y_{k}\right)$ that

$$
\left(x_{k}^{*}, y_{k}^{*},-1\right) \in \widehat{N}\left(\left(x_{k}, y_{k}, \alpha_{k}\right) ; \Omega_{1} \cap \Omega_{2}\right) \text { with } \alpha_{k}:=g\left(x_{k}, y_{k}\right)=\varphi_{\Theta-\bar{y}, \mathbf{k}}\left(y_{k}\right) .
$$

We will work on the Asplund space $X \times Y \times \mathbb{R}$ with the sum norm and its dual space $X^{*} \times Y^{*} \times \mathbb{R}$ with the max norm. We apply Lemma 4.1 for each $k \in \mathbb{N}$ to choose sequences

$$
\begin{aligned}
& \lambda_{k} \geq 0,\left(x_{i k}, y_{i k}, \alpha_{i k}\right) \in \Omega_{i}(i=1,2), \\
& \left(\mathbf{0}, y_{1 k}^{*}, t_{1 k}\right) \in \widehat{N}\left(\left(x_{1 k}, y_{1 k}, \alpha_{1 k}\right) ; \Omega_{1}\right)=\{0\} \times \widehat{N}\left(\left(y_{1 k}, \alpha_{1 k}\right) ; \operatorname{epi} \varphi_{\Theta-\bar{y}, \mathbf{k}}\right), \\
& \left(x_{2 k}^{*}, y_{2 k}^{*}, 0\right) \in \widehat{N}\left(\left(x_{2 k}, y_{2 k}, \alpha_{2 k}\right) ; \Omega_{2}\right)=\widehat{N}\left(\left(x_{2 k}, y_{2 k}\right) ; \operatorname{gph} F\right) \times\{0\}
\end{aligned}
$$

satisfying

$$
\begin{gathered}
\left\|\left(x_{i k}, y_{i k}, \alpha_{i k}\right)-\left(x_{k}, y_{k}, \alpha_{k}\right)\right\| \leq \varepsilon_{k}, i \in\{1,2\}, \\
\left\|\left(\mathbf{0}, y_{1 k}^{*}, t_{1 k}\right)+\left(x_{2 k}^{*}, y_{2 k}^{*}, 0\right)-\lambda_{k}\left(x_{k}^{*}, y_{k}^{*},-1\right)\right\| \leq 2 \varepsilon_{k}, \\
1-\varepsilon_{k} \leq \max \left\{\lambda_{k},\left\|y_{1 k}^{*}\right\|,\left|t_{1 k}\right|\right\} \leq 1+\varepsilon_{k} .
\end{gathered}
$$


Since $\left\|x_{k}^{*}\right\| \rightarrow 0$ and $\left\|y_{k}^{*}\right\| \rightarrow 0$ (see (4.8)), we get from (4.9) and (4.10) that the sequences $\left\{y_{1 k}^{*}\right\},\left\{t_{1 k}\right\}$, $\left\{x_{2 k}^{*}\right\},\left\{y_{2 k}^{*}\right\}$, and $\left\{\lambda_{k}\right\}$ are bounded. Taking into account the Asplund property of $X$ and $Y$, we may suppose that

$$
\left(y_{1 k}^{*}, x_{2 k}^{*}, y_{2 k}^{*}\right) \stackrel{w^{*}}{\rightarrow}\left(y^{*}, x^{*},-y^{*}\right), \lambda_{k} \rightarrow \bar{\lambda} \text { and } t_{1 k} \rightarrow \bar{\lambda} .
$$

We claim that $\bar{\lambda} \neq 0$. Arguinging by contradition, assume that $\bar{\lambda}=0$. We obtain (4.9) from that $\left\|x_{2 k}^{*}\right\| \rightarrow$ 0 . By the structure of the sets $\Omega_{1}$ and $\Omega_{2}$ we get $y^{*} \in \partial^{\infty} \varphi_{\Theta-\bar{y}, \mathbf{k}}(\bar{y})$ and $-y^{*} \in D_{M}^{*} F^{-1}(\bar{y}, \bar{x})(\mathbf{0})$, i.e.,

$$
-y^{*} \in D_{M}^{*} F^{-1}(\bar{y}, \bar{x})(\mathbf{0}) \cap\left(-\partial^{\infty} \varphi_{\Theta-\bar{y}, \mathbf{k}}(\bar{y})\right) .
$$

The subdifferential of the scalarization functional $\varphi_{\Theta-\bar{y}, \mathbf{k}}$ in Theorem $3.1 \partial^{\infty} \varphi_{\Theta-\bar{y}, \mathbf{k}}(\bar{y})=H_{0}(\mathbf{k}) \cap(-N(\mathbf{0} ; \Theta))$ and the imposed mixed qualification condition for $\{F, \Theta\}$ ensure $y^{*}=\mathbf{0}$. We consider two cases according to the SNC assumptions.

Case 1: Assume that $\Theta$ is SNC at $\mathbf{0}$. Then, we get from $y_{1 k}^{*} \stackrel{w^{*}}{\rightarrow} y^{*}$ and $y^{*}=\mathbf{0}$ that $\left\|y_{1 k}^{*}\right\| \rightarrow 0$.

Case 2: Assume that $F^{-1}$ is PSNC at $(\bar{y}, \bar{x})$. Then, we get from $\left\|x_{2 k}^{*}\right\| \rightarrow 0$ and $y_{2 k}^{*} \stackrel{w^{*}}{\rightarrow} \mathbf{0}$ that $\left\|y_{2 k}^{*}\right\| \rightarrow 0$. Taking into account (4.9) and $\left\|y_{k}^{*}\right\| \rightarrow 0$, we get $\left\|y_{1 k}^{*}\right\| \rightarrow 0$.

In both cases, we obtain $\left\|y_{1 k}^{*}\right\| \rightarrow 0$. This together with $\lambda_{k} \rightarrow 0, t_{1 k} \rightarrow \bar{\lambda}=0$ contradicts the nontriviality condition (4.10) and thus verifies $\bar{\lambda}>0$. Without loss of generality, we may assume that $\lambda_{k} \equiv 1$ for all $k \in \mathbb{N}$. We now have

$$
y^{*} \in \partial \varphi_{\Theta-\bar{y}, \mathbf{k}}(\bar{y}) \text { and }-y^{*} \in D_{M}^{*} F^{-1}(\bar{y}, \bar{x})(\mathbf{0}) .
$$

Since $D_{M}^{*} F^{-1}(\bar{y}, \bar{x})(\mathbf{0}) \subseteq D_{N}^{*} F^{-1}(\bar{y}, \bar{x})(\mathbf{0})$, we get

$$
-y^{*} \in D_{N}^{*} F^{-1}(\bar{y}, \bar{x})(\mathbf{0}) \Leftrightarrow\left(\mathbf{0},-y^{*}\right) \in N((\bar{x}, \bar{y}), \operatorname{gph} F) \Leftrightarrow 0 \in D_{N}^{*} F(\bar{x}, \bar{y})\left(y^{*}\right) .
$$

By the subdifferential estimates of the scalarization function $\varphi_{\Theta-\bar{y}, \mathbf{k}}$ in Theorem 3.1, we have $y^{*} \in$ $-N(-\bar{y} ; \Theta-\bar{y})=-N(\mathbf{0} ; \Theta)$ and $y^{*}(\mathbf{k})=1$ and thus completes the proof of the theorem.

\section{Remark 4.1. (on assumptions of Theorem 4.1).}

(a) Analyzing the proof of Theorem 4.1, we observe that the mixed qualification condition for $\{F, \Theta\}$ in (4.6) could be replaced by the qualification condition for $\left\{F, \varphi_{\Theta-\bar{y}, \mathbf{k}}\right\}$

$$
\left.D_{M}^{*} F^{-1}(\bar{y}, \bar{x})(\mathbf{0}) \cap\left(-\partial^{\infty} \varphi_{\Theta-\bar{y}, \mathbf{k}}\right)(\bar{y})\right)=\{\mathbf{0}\} .
$$

and that the choice of $y^{*}$ may be chosen from $\partial \varphi_{\Theta-\bar{y}, \mathbf{k}}(\bar{y})$.

(b) The mixed qualification condition (4.6) for $\{F, \Theta\}$ is satisfied when one of the following conditions holds:

- $\Theta$ is a solid and convex cone;

- $F^{-1}$ is locally Lipschitz-like around $(\bar{y}, \bar{x})$.

In [8], Durea and Strugariu derived a fuzzy result based on the characteristic of openness property at Pareto minimizers with respect to convex ordering cones since if $(\bar{x}, \bar{y}) \in \mathrm{gph} F$ is a local Pareto minimizer of $F$ and $K$ is not a linear subspace of $Y$; i.e. $K \backslash(-K) \neq \emptyset$, then the set-valued map $F$ is not open at $(\bar{x}, \bar{y})$. Recall that $F$ is said to be open at $(\bar{x}, \bar{y}) \in \operatorname{gph} F$ if the image through $F$ of every neighborhood of $x$ is a neighborhood of $y$. The next corollary establishes a connection between $\Theta$-minimality and linear openness. 
Corollary 4.1. (Lipschitz-like behavior at $\Theta$-minimal solutions of set-valued mappings). Assume that all assumptions in Theorem 4.1 are satisfied. If $(\bar{x}, \bar{y})$ is a $\Theta$-minimal solution of $F$, then $F^{-1}$ is not Lipschitz-like around $(\bar{y}, \bar{x})$ and thus $F$ is not linearly open at $(\bar{x}, \bar{y})$.

Proof. Theorem 4.1 ensures that

$$
D_{M}^{*} F^{-1}(\bar{y}, \bar{x})(\mathbf{0}) \neq\{\mathbf{0}\} .
$$

Therefore, $F^{-1}$ is not Lipschitz-like around $(\bar{y}, \bar{x})$ due to Lemma 2.6.

It is well known that a constrained optimization problem (4.1)

$$
\Theta \text {-Minimize } F(x) \text { subject to } x \in \Omega \text {, }
$$

could be converted into an equivalent unconstrained problem by considering the restricted mapping $F_{\Omega}$ : $X \rightrightarrows Y$ of $F$ over $\Omega$, where $F_{\Omega}$ is defined by

$$
F_{\Omega}(x):=\left\{\begin{array}{lll}
F(x), & \text { if } & x \in \Omega \\
\emptyset, & \text { if } & x \notin \Omega
\end{array}\right.
$$

Before establishing necessary conditions for $\Theta$-minimal solutions to constrained optimization problems, we study several properties for the restricted mapping $F_{\Omega}$.

Proposition 4.2. (calculus rules for restricted mappings). Let $F: X \rightrightarrows Y$ be a set-valued mapping acting between Aspund spaces, $\Omega$ be a nonempty subset of $X$ and $F_{\Omega}$ be the restricted mapping of $F$ over $\Omega$. Given $\bar{x} \in \Omega \cap \operatorname{dom} F$ and $\bar{y} \in F(\bar{x})$. Assume that $\operatorname{gph} F$ and $\Omega$ are locally closed around $(\bar{x}, \bar{y})$ and $\bar{x}$, respectively. Then:

(i) (coderivative rule for $F_{\Omega}$ ) Assume that the norm-convergence qualification condition for $\{F, \Omega\}$ is satisfied: for any sequence $\left(x_{1 k}, x_{2 k}, y_{1 k}, x_{1 k}^{*}, x_{2 k}^{*}, y_{1 k}^{*}\right)$ satisfying

$$
\left[\begin{array}{l}
\left(x_{1 k}, y_{1 k}\right) \in \operatorname{gph} F, x_{2 k} \in \Omega, x_{1 k}^{*} \in \widehat{D}^{*} F\left(x_{1 k}, y_{1 k}\right)\left(y_{1 k}^{*}\right), x_{2 k}^{*} \in \widehat{N}\left(x_{2 k} ; \Omega\right), \\
\left(x_{1 k}, y_{1 k}\right) \rightarrow(\bar{x}, \bar{y}), x_{2 k} \rightarrow \bar{x},\left(x_{1 k}^{*}, x_{2 k}^{*}\right) \stackrel{w^{*}}{\longrightarrow}\left(x_{1}^{*}, x_{2}^{*}\right)
\end{array}\right]
$$

one has

$$
\left(\left\|x_{1 k}^{*}+x_{2 k}^{*}\right\| \rightarrow 0 \wedge\left\|y_{k}^{*}\right\| \rightarrow 0\right) \Rightarrow\left\|x_{1 k}^{*}\right\|+\left\|x_{2 k}^{*}\right\| \rightarrow 0 .
$$

Then, the coderivative of $F_{\Omega}$ can be estimated by

$$
\forall y^{*} \in Y^{*}: D^{*} F_{\Omega}(\bar{x}, \bar{y})\left(y^{*}\right) \subseteq D^{*} F(\bar{x}, \bar{y})\left(y^{*}\right)+N(\bar{x} ; \Omega),
$$

where $D^{*}$ stand for both the normal and mixed coderivatives defined in Definition 2.4.

(ii) (PSNC rule for $F_{\Omega}$ ) Assume that the following conditions hold:

(a) (SNC condition) either $F$ is SNC at $(\bar{x}, \bar{y})$, or $F^{-1}$ is PSNC at $(\bar{y}, \bar{x})$ and $\Omega$ is SNC at $\bar{x}$.

(b) (mixed qualification condition for $\{F, \Omega\}$ ) for any sequence $\left(x_{1 k}, x_{2 k}, y_{1 k}, x_{1 k}^{*}, x_{2 k}^{*}, y_{1 k}^{*}\right)$ satisfying condition (4.11), one has

$$
\left(\left\|x_{1 k}^{*}+x_{2 k}^{*}\right\| \rightarrow 0 \wedge y_{k}^{*} \stackrel{w^{*}}{\rightarrow} \mathbf{0}\right) \Rightarrow x_{1}^{*}=x_{2}^{*}=\mathbf{0},
$$

where $x_{i}^{*}$ is the weak ${ }^{*}$-limit of the sequence $\left\{x_{i k}^{*}\right\}$ for $i=1,2$. Then, the mapping $F_{\Omega}^{-1}$ is PSNC at $(\bar{y}, \bar{x})$. 
Proof. We will prove the PSNC property for $F_{\Omega}^{-1}$ in (ii) and refer the reader to [20, Proposition 3.12] for a proof of (i).

Since gph $F_{\Omega}=\Omega_{1} \cap \Omega_{2}$ where $\Omega_{1}$ and $\Omega_{2}$ are subsets in the product space $X \times Y$ defined by

$$
\Omega_{1}:=\operatorname{gph} F \text { and } \Omega_{2}:=\Omega \times Y .
$$

To justify the PSNC property of $F_{\Omega}^{-1}$, one needs to show that for any sequences $\left(x_{k}, y_{k}\right) \in \Omega_{1} \cap \Omega_{2}$, $\left(x_{k}^{*}, y_{k}^{*}\right) \in \widehat{N}\left(\left(x_{k}, y_{k}\right) ; \Omega_{1} \cap \Omega_{2}\right)$, for all $k \in \mathbb{N}$, if $\left(x_{k}, y_{k}\right) \rightarrow(\bar{x}, \bar{y}),\left\|x_{k}^{*}\right\| \rightarrow 0, y_{k}^{*} \stackrel{w^{*}}{\rightarrow} 0$, then $\left\|y_{k}^{*}\right\| \rightarrow 0$ as $k \rightarrow \infty$. Since we are dealing with arbitrary sequences satisfying the above convergence properties, it is sufficient to show that $\left\|y_{k}^{*}\right\| \rightarrow 0$ along a subsequence.

We fix a sequence $\varepsilon_{k} \downarrow 0$ and apply [20, Lemma 3.1] for each $k \in \mathbb{N}$. In this way, we find

$$
\begin{aligned}
& \lambda_{k} \geq 0,\left(x_{i k}, y_{i k}\right) \in \Omega_{i},\left\|\left(x_{i k}, y_{i k}\right)-\left(x_{k}, y_{k}\right)\right\| \leq \varepsilon_{k},(i=1,2), \\
& \left(x_{1 k}^{*}, y_{1 k}^{*}\right) \in \widehat{N}\left(\left(x_{1 k}, y_{1 k}\right) ; \Omega_{1}\right)=\widehat{N}\left(\left(x_{1 k}, y_{1 k}\right) ; \operatorname{gph} F\right) \\
& \left(x_{2 k}^{*}, \mathbf{0}\right) \in \widehat{N}\left(\left(x_{2 k}, y_{2 k} ; \Omega_{2}\right)=\widehat{N}\left(x_{2 k} ; \Omega\right) \times\{\mathbf{0}\},\right.
\end{aligned}
$$

satisfying

$$
\begin{gathered}
\left\|\left(x_{1 k}^{*}, y_{1 k}^{*}\right)+\left(x_{2 k}^{*}, \mathbf{0}\right)-\lambda_{k}\left(x_{k}^{*}, y_{k}^{*}\right)\right\| \leq 2 \varepsilon_{k}, \\
1-\varepsilon_{k} \leq \max \left\{\lambda_{k},\left\|x_{1 k}^{*}\right\|,\left\|y_{1 k}^{*}\right\|\right\} \leq 1+\varepsilon k .
\end{gathered}
$$

Since $\left\|x_{k}^{*}\right\| \rightarrow 0$ and $y_{k}^{*} \stackrel{w^{*}}{\rightarrow} \mathbf{0}$, the sequence $\left(x_{k}^{*}, y_{k}^{*}\right)$ is bounded. We get from (4.15) and (4.16) that the sequences $\left\{\left(x_{1 k}^{*}, y_{1 k}^{*}, x_{2 k}^{*}\right)\right\}$ and $\left\{\lambda_{k}\right\}$ are bounded. Taking into account that the Asplund property of the spaces $X$ and $Y$, we may suppose that the triple sequence weak ${ }^{*}$ converges to some $\left(x_{1}^{*}, y_{1}^{*} x_{2}^{*}\right)$, and that $\lambda_{k} \rightarrow \bar{\lambda} \geq 0$ as $k \rightarrow \infty$. This implies, by (4.15) and by the convergence of $\left(x_{k}^{*}, y_{k}^{*}\right)$, that

$$
\left\|x_{1 k}^{*}+x_{2 k}^{*}\right\| \rightarrow 0 \text { and } y_{1 k}^{*} \stackrel{w^{*}}{\rightarrow} \mathbf{0} .
$$

The imposed mixed qualification condition for $\{F, \Omega\}$ forces $x_{1}^{*}=x_{2}^{*}=\mathbf{0}$ and $y_{1}^{*}=\mathbf{0}$.

We now consider two cases according to the SNC conditions imposed in the theorem.

Case 1: Assume that $F$ is SNC at $(\bar{x}, \bar{y})$; i.e., $\operatorname{gph} F$ is SNC at $(\bar{x}, \bar{y})$. Then, we have $\left\|x_{1 k}^{*}\right\| \rightarrow 0$ and $\left\|y_{1 k}^{*}\right\| \rightarrow 0$ as $k \rightarrow \infty$.

Case 2: Assume that $\Omega$ is SNC at $\bar{x}$. It follows that $\left\|x_{2 k}^{*}\right\| \rightarrow 0$ and hence $\left\|x_{1 k}^{*}\right\| \rightarrow 0$ as $k \rightarrow \infty$. Then, the PSNC of $F^{-1}$ ensures that $\left\|y_{1 k}^{*}\right\| \rightarrow 0$ as $k \rightarrow \infty$.

In both cases, we obtained $\left\|x_{1 k}^{*}\right\| \rightarrow 0$ and $\left\|y_{1 k}^{*}\right\| \rightarrow 0$ as $k \rightarrow \infty$. Therefore, the nontriviality condition (4.16) implies $\lambda_{k} \rightarrow \bar{\lambda}=1$ as $k \rightarrow \infty$, and (4.15) yields $\left\|y_{k}^{*}\right\| \rightarrow 0$ as $k \rightarrow \infty$. The proof of (ii) is complete.

Remark 4.2. If the pair $\{F, \Omega\}$ satisfies both SNC and qualification conditions in (ii), then the normconvergence qualification condition in (i) is fulfilled. This means that under the assumptions imposed in (ii), we could use the coderivative rule for $F_{\Omega}$.

Theorem 4.2. (necessary conditions for $\Theta$-minimal solutions). Consider problem (4.1) and a $\Theta$ minimal solution $(\bar{x}, \bar{y})$. Let $\mathbf{k} \in \operatorname{dir}(\Theta)$ be a scalarization direction of $\Theta$, and $\varphi=\varphi_{\Theta-\bar{y}, k}$ be the scalarization functional defined in Definition 2.2. Assume that the following conditions hold:

(H1) (closedness condition) the domination set $\Theta$ is locally closed around the origin, $\operatorname{gph} F$ is closed around $(\bar{x}, \bar{y})$, and $\Omega$ is locally closed around $\bar{x}$.

(H2) (SNC conditions) One of the following conditions holds: 
(a) $\Theta$ is $S N C$ at $\mathbf{0}$ and $\Omega$ is $S N C$ at $\bar{x}$;

(b) $\Theta$ is SNC at $\mathbf{0}$ and $F$ is PSNC at $(\bar{x}, \bar{y})$;

(c) $\Omega$ is SNC at $\bar{x}$ and $F^{-1}$ is PSNC at $(\bar{y}, \bar{x})$;

(d) $F$ is SNC at $(\bar{x}, \bar{y})$.

(H3) (qualification conditions)

- Either the norm-convergence qualification condition for $\{F, \Omega\}$ is satisfied for the SNC condition (a) or (b), or the mixed one is fulfilled for the SNC condition (c) or (d).

- The qualification condition for $\{\Theta, F, \Omega\}$ : for any sequence

$$
\left\{\left(x_{1 k}, x_{2 k}, y_{1 k}, x_{1 k}^{*}, x_{2 k}^{*}, y_{1 k}^{*}\right)\right\}
$$

satisfying condition (4.11) one has

$$
\left[\begin{array}{c}
x_{1 k}^{*} \stackrel{w^{*}}{\rightarrow} x_{1}^{*}, x_{2 k}^{*} \stackrel{w^{*}}{\rightarrow} x_{2}^{*},\left\|x_{1 k}^{*}+x_{2 k}^{*}\right\| \rightarrow 0, \\
y_{1 k}^{*} \stackrel{w^{*}}{\rightarrow}-y_{1}^{*}, y_{1}^{*} \in-N(\mathbf{0} ; \Theta) \cap H_{0}(\mathbf{k})
\end{array}\right] \Rightarrow\left[\begin{array}{c}
x_{1}^{*}=x_{2}^{*}=\mathbf{0} \\
y_{1}^{*}=\mathbf{0}
\end{array}\right]
$$

Then, there is $y^{*} \in-N(\mathbf{0} ; \Theta)$ with $y^{*}(\mathbf{k})=1$ satisfying

$$
\mathbf{0} \in \partial F(\bar{x}, \bar{z})\left(\bar{y}^{*}\right)+N(\bar{x} ; \Omega) .
$$

Proof. Since $(\bar{x}, \bar{y})$ be a $\Theta$-minimal solution to problem (4.1), it is a $\Theta$-minimal solution of the mapping $F_{\Omega}$. Under the assumptions made, we could find some $y^{*} \in \partial \varphi_{\Theta-\bar{y}, \mathbf{k}}(\bar{y})=H_{1}(\mathbf{k}) \cap(-N(\varphi(\bar{y}) \mathbf{k}-\bar{y} ; \Theta))$ such that $-y^{*} \in D_{M}^{*} F^{-1}(\bar{y}, \bar{x})(\mathbf{0})$ provided that the following SNC and qualification conditions hold:

- (SNC condition) either $\Theta$ is SNC at $\mathbf{0}$ or $F_{\Omega}^{-1}$ is PSNC at $(\bar{y}, \bar{x})$.

- (qualification condition) $D_{M}^{*} F_{\Omega}^{-1}(\bar{y}, \bar{x})(\mathbf{0}) \cap-\partial^{\infty} \varphi_{\Theta-\bar{y}, \mathbf{k}}(\bar{y})=\{\mathbf{0}\}$.

Note that $D_{M}^{*} F_{\Omega}^{-1}(\bar{y}, \bar{x})(\mathbf{0}) \subseteq D^{*} F_{\Omega}^{-1}(\bar{y}, \bar{x})(\mathbf{0})$. We get from $-y^{*} \in D^{*} F^{-1}(\bar{y}, \bar{x})(\mathbf{0})$ that $\left(0,-y^{*}\right) \in$ $N\left((\bar{x}, \bar{y}) ; \operatorname{gph} F_{\Omega}\right)$ and thus $\mathbf{0} \in D_{N}^{*} F_{\Omega}(\bar{x}, \bar{y})\left(y^{*}\right)$. Since the mixed qualification condition for $\{F, \Omega\}$ together with the qualification condition (c) or (d) implies the norm-convergence qualification condition for $\{F, \Omega\}$. Employing the coderivative rule for $F_{\Omega}$, we arrive at

$$
0 \in D_{N}^{*} F(\bar{x}, \bar{y})\left(y^{*}\right)+N(\bar{x} ; \Omega)
$$

and thus completes the proof of the theorem provided that the aforementioned SNC and qualification conditions are satisfied.

By Proposition 4.2, the SNC condition is fulfilled under the assumptions made in the theorem.

To check the qualification condition, take an arbitrary element

$$
y^{*} \in D_{M}^{*} F_{\Omega}^{-1}(\bar{y}, \bar{x})(\mathbf{0}) \cap\left(-\partial^{\infty} \varphi_{\Theta-\bar{y}, \mathbf{k}}(\bar{y})\right) .
$$

Using the definition of mixed coderivative, we could find sequences $\left\{\left(x_{k}, y_{k}, x_{k}^{*}, y_{k}^{*}\right)\right\}$ satisfying $\left(x_{k}, y_{k}\right) \in$ $\operatorname{gph} F\left(x_{k}^{*}, y_{k}^{*}\right) \in \widehat{N}\left(\left(x_{k}, y_{k}\right) ; \operatorname{gph} F\right),\left(x_{k}, y_{k}\right) \rightarrow(\bar{x}, \bar{y})$,

$$
\left\|x_{k}^{*}\right\| \rightarrow 0 \text { and } y_{k}^{*} \stackrel{w^{*}}{\rightarrow} y^{*} .
$$


Since gph $F_{\Omega}=\Omega_{1} \cap \Omega_{2} \subseteq X \times Y$ with $\Omega_{1}=\operatorname{gph} F$ and $\Omega_{2}=\Omega \times Y$. We fix a sequence $\varepsilon_{k} \downarrow 0$ and apply the fuzzy intersection rule [20, Lemma 3.1] for each $k \in \mathbb{N}$. In this way, we could choose sequences

$$
\begin{aligned}
& \lambda_{k} \geq 0,\left(x_{i k}, y_{i k}\right) \in \Omega_{i},\left\|\left(x_{i k}, y_{i k}\right)-\left(x_{k}, y_{k}\right)\right\| \leq \varepsilon_{k},(i=1,2), \\
& \left(x_{1 k}^{*}, y_{1 k}^{*}\right) \in \widehat{N}\left(\left(x_{1 k}, y_{1 k}\right) ; \Omega_{1}\right)=\widehat{N}\left(\left(x_{1 k}, y_{1 k}\right) ; \operatorname{gph} F\right), \\
& \left(x_{2 k}^{*}, \mathbf{0}\right) \in \widehat{N}\left(\left(x_{2 k}, y_{2 k} ; \Omega_{2}\right)=\widehat{N}\left(x_{2 k} ; \Omega\right) \times\{\mathbf{0}\},\right.
\end{aligned}
$$

satisfying

$$
\begin{gathered}
\left\|\left(x_{1 k}^{*}, y_{1 k}^{*}\right)+\left(x_{2 k}^{*}, \mathbf{0}\right)-\lambda_{k}\left(x_{k}^{*}, y_{k}^{*}\right)\right\| \leq 2 \varepsilon_{k}, \\
1-\varepsilon_{k} \leq \max \left\{\lambda_{k},\left\|x_{1 k}^{*}\right\|,\left\|y_{1 k}^{*}\right\|\right\} \leq 1+\varepsilon k .
\end{gathered}
$$

Since $\left\|x_{k}^{*}\right\| \rightarrow 0$ and $y_{k}^{*} \stackrel{w^{*}}{\rightarrow} \mathbf{0}$ (see (4.18)), the sequences $\left\{x_{k}^{*}\right\}$ and $\left\{y_{k}^{*}\right\}$ are bounded. We get from (4.19) and (4.20) that the sequences $\left\{\left(x_{1 k}^{*}, y_{1 k}^{*}, x_{2 k}^{*}\right)\right\}$ and $\{k\}$ are bounded. Taking into account the Asplund property of the spaces $X$ and $Y$, we may suppose that the triple sequence weak*-converge to some $\left(x^{*}, y^{*},-x^{*}\right)$, and that $\lambda_{k} \rightarrow \bar{\lambda} \geq 0$ as $k \rightarrow \infty$. We will show that $\bar{\lambda}>0$. Arguing by contradiction, we assume that $\bar{\lambda}=0$. Then, we get

$$
y_{1 k}^{*} \stackrel{w^{*}}{\rightarrow} \mathbf{0} \text { and }\left\|x_{1 k}^{*}+x_{2 k}^{*}\right\| \rightarrow 0 .
$$

The mixed limiting qualification condition yields $x^{*}=\mathbf{0}$ and $y^{*}=\mathbf{0}$. Each qualification condition in (a)-(d) leads to $\left\|x_{1 k}^{*}\right\| \rightarrow 0,\left\|x_{2 k}^{*}\right\| \rightarrow 0$ and $\left\|y_{k}^{*}\right\| \rightarrow 0$ contradicting to the nontriviality condition (4.20). Therefore, $\bar{\lambda}>0$. Without loss of generality, we may assume that $\lambda_{k} \equiv 1$ for all $k \in \mathbb{N}$ in (4.19). We now have

$$
y_{1 k}^{*} \stackrel{w^{*}}{\rightarrow} y^{*} \text { and }\left\|x_{1 k}^{*}+x_{2 k}^{*}\right\| \rightarrow 0 .
$$

The qualification condition for $\left\{\varphi_{\Theta-\bar{y}, F, \Omega}\right\}$ forces $y^{*}=\mathbf{0}$. This justifies the fulfillment of the qualitication condition.

\section{Remark 4.3. (on local minimal solutions).}

(a) It is important to emphasize that the results obtained in this section holds for fully locallized $\Theta$-minimal solutions of problem (4.1) since if $(\bar{x}, \bar{y})$ is such a minimal solution, $\bar{x} \in \operatorname{dom} F$, $\bar{y} \in F(\bar{x})$, and there exist a neighborhood of $U$ of $\bar{x}$ and a neighborhood $V$ of $\bar{y}$ such that $(F(\Omega \cap$ $\operatorname{cl} U) \cap \operatorname{cl} V) \cap(\bar{y}-\Theta)=\{\bar{y}\}$; i.e., it is a $\Theta$-minimal solution of the set-valued mapping $\widetilde{F}$ over $\Omega$, where $\widetilde{F}: X \rightrightarrows Y$ is define by $\operatorname{gph} \widetilde{F}=\operatorname{gph} F \cap(\operatorname{cl}(U) \times \operatorname{cl}(V))$ and thus $D_{N}^{*} F(\bar{x}, \bar{y})\left(y^{*}\right)=$ $D_{N}^{*} \widetilde{F}(\bar{x}, \bar{y})\left(y^{*}\right), D_{M}^{*} F(\bar{x}, \bar{y})(\mathbf{0})=D_{M}^{*} \widetilde{F}(\bar{x}, \bar{y})(\mathbf{0})$ and $D_{N}^{*} F^{-1}(\bar{y}, \bar{x})\left(y^{*}\right)=D_{N}^{*} \widetilde{F}^{-1}(\bar{y}, \bar{x})(\mathbf{0})$.

(b) Assume, in addition, that $\Theta+\Theta \subseteq \Theta$. Then, we could formulate subdifferential versions of necessary optimality conditions for $\Theta$-minimal solutions as in $[1,4]$ since if $(\bar{x}, \bar{y})$ is a $\Theta$-minimal solution of a set-valued mapping $F$, then it is also a $\Theta$-minimal solution of the epigraphical multifunction of $F$ with respect to $\Theta$. Let it formulate the counterpart of Theorem 4.1.

Corollary 4.2. (subdifferential necessary conditions for $\Theta$-minimal solutions of set-valued mappings). Consider problem (4.1) and a $\Theta$-minimal solution $(\bar{x}, \bar{y})$. Let $\mathbf{k} \in \operatorname{dir}(\Theta)$ be a scalarization direction of $\Theta$, and $\varphi=\varphi_{\Theta-\bar{y}, k}$ be the scalarization functional defined in Definition 2.2. Assume that $\Theta+\Theta \subseteq \Theta$ and $\Theta \cap(-\Theta)=\{\mathbf{0}\}$. Assume also that

(H1) (closedness condition) The domination set $\Theta$ is locally closed around the origin and epi $F=$ $\operatorname{gph} \mathscr{E}_{F}$ is locally closed around $(\bar{x}, \bar{y})$. 
(H2) (PSNC condition) Either $\Theta$ is SNC at $\mathbf{0}$ or $\mathscr{E}_{F}^{-1}$ is PSNC at $(\bar{y}, \bar{x})$.

(H3) (mixed qualification condition for $\left\{\mathscr{E}_{F}, \Theta\right\}$ )

$$
\left(y^{*} \in D_{M^{*}}^{*} \mathscr{E}^{-1}(\bar{y}, \bar{x})(\mathbf{0}) \cap(-N(\mathbf{0} ; \Theta)) \wedge y^{*}(\mathbf{k})=0\right) \Rightarrow y^{*}=\mathbf{0} .
$$

Then, there is $y^{*} \in-N(\mathbf{0} ; \Theta)$ with $y^{*}(\mathbf{k})=1$ satisfying

$$
\mathbf{0} \in \partial F(\bar{x}, \bar{y})\left(y^{*}\right) .
$$

Proof. By Theorem 4.1, it is sufficient to justify that $(\bar{x}, \bar{y})$ is a $\Theta$-minimal solution of $\mathscr{E}_{F}$. Arguing by contradiction, assume that it is not a $\Theta$-minimal solution of $\mathscr{E}_{F}$. There exists $(x, y) \in$ epi $\mathscr{E}_{F}$ with $y \neq \bar{y}$ and $y \leq_{\Theta} \bar{y}$, i.e., $y \in \bar{y}-\Theta$. By the definition of epigraph, there is $\theta \in \Theta$ such that $\tilde{y}=y-\theta \in F(x)$. Since $y \in \bar{y}-\Theta, \tilde{y}=y-\theta \in \bar{y}-\Theta-\theta \subseteq \bar{y}-\Theta$, i.e., $\tilde{y} \leq_{\Theta} \bar{y}$. By the $\Theta$-minimality of $(\bar{x}, \bar{y})$ to $F$, we have $\tilde{y}=y-\theta=\bar{y}$ and thus $y \in \bar{y}+\Theta$. The pointedness of $\Theta$ ensures that $y=\bar{y}$ contradicting to the choice of $y(y \neq \bar{y})$. The proof is complete.

\section{APPLICATIONS IN APPROXIMATION THEORY}

As an application of our results, we present some optimality conditions for set-valued (not necessary convex) approximation problems having a practical importance described in [15].

We assume that $X, Y$ and $Z$ are real Banach spaces, $\Theta$ is a nontrivial, closed, convex and pointed cone in $Y$. In order to formulate the vector control approximation problem, let us introduce a vector-valued norm (see [16]) as an application $\|\cdot\|: Z \rightarrow \Theta$ which for all $z, z_{1}, z_{2} \in Z$ and for all $\lambda \in \mathbb{R}$ satisfies:

$$
\begin{aligned}
& \|z \mid\|=0 \Longleftrightarrow z=0 ; \\
& \|\lambda z\|=|\lambda|\|z\| ; \\
& \left\|z_{1}+z_{2}\right\| \in\left\|z_{1}\right\|\left|+\left\|z_{2}\right\|\right|-\Theta .
\end{aligned}
$$

A subdifferential (denoted $\partial \leq$ ) for vector-valued functions was proposed by Jahn in [16] and for the particular case of the above vector-valued norm $\|\cdot \mid\|$ it has the following form:

$$
\partial^{\leq}\|\cdot\|\left(z_{0}\right)=\left\{T \in L(Z, Y) \mid T\left(z_{0}\right)=\left\|z_{0}\right\| \wedge(\forall z \in Z:\|z\|-T(z) \in \Theta)\right\},
$$

where $L(Z, Y)$ denotes the space of linear continuous operators from $Z$ into $Y$.

Furthermore, we assume that $\partial \leq|\|\cdot\|| \neq \emptyset$. Sufficient conditions for $\partial \leq\|\cdot\| \| \emptyset$ are given by Jahn [16] (for instance, \|\|$\cdot \|$ is continuous and $\Theta$ has the Daniell property which means that every decreasing net (i.e., $i \leq j$ implies $x_{j} \leq x_{i}$ ) having a lower bound converges to its infimum).

Note that for every $\Theta_{+} \in \Theta^{*}$, the mapping $y^{*}(\||\cdot|\|)$ is convex, hence its subdifferential is understood in the sense of convex analysis.

Assuming additionally that $\Theta$ has a weakly compact base, we adapt a result by Valadier [23] which is useful in the sequel.

Lemma 5.1. ([23]). Let $(Z,\|\cdot\|)$ and $(Y,\|\cdot\|)$ be real reflexive Banach spaces and $\Theta \subseteq Y$ a proper convex Daniell cone with a weakly compact base. Suppose that the vector-valued norm $\|\cdot|\||$ is continuous. Then for every $y \in Y$ and $y^{*} \in \Theta^{*}$ one has

$$
y^{*}\left(\partial^{\leq}\|\cdot \mid\|(y)\right)=\partial\left(y^{*}(\||\|\mid\|))(y) .\right.
$$


Lemma 5.2. ([6, Corollary 2.4]). Let $f: X \rightarrow Y$ be convex with $\Theta$ normal. Then, if $f$ is continuous at $x_{0}$ and $X$ and $Y$ are normed, then it is locally Lipschitz throughout the algebraic interior of $\operatorname{dom} f$.

Suppose now that $W$ is a nonempty set and the cost function $g: X \times W \rightarrow Y$ is locally Lipschitz, $A_{i} \in L(X, Z)$ and $\alpha_{i} \geq 0(i=1, \ldots, n)$. In the following, $A_{i}^{*}$ denotes the adjoint operator to $A_{i}$. Then, we consider for $x \in \Omega \subseteq X$ and $a^{i} \in Z(i=1, \ldots, n)$ the vector-valued approximation problem

$$
\text { minimize } f(x, w):=g(x, w)+\sum_{i=1}^{n} \alpha_{i}\left\|A_{i}(x)-a^{i}\right\| \text { subject to } x \in \Omega,
$$

where $w \in W(x)$ stands for the control parameter and "minimization" is understood with respect to the partial order generated by a nontrivial, closed, convex and pointed cone $\Theta \subseteq Y$ in (2.1) and $\Omega \subseteq X$ is closed. By considering set-valued mappings $G(x):=\{g(x, w) \mid w \in W(x)\}$, the vector-valued approximation problem (5.2) is equivalent to the following set-valued approximation problem

$$
\text { minimize } F(x):=G(x)+\left\{\sum_{i=1}^{n} \alpha_{i}\left\|A_{i}(x)-a^{i}\right\|\right\} \text { subject to } x \in \Omega \text {. }
$$

Theorem 5.1. Suppose that $X, Y, Z$ are reflexive Banach spaces, $\Theta \subseteq Z$ a proper convex Daniell cone with a weakly compact base, $|\|\cdot\|| \|$ is continuous and $\Omega$ is a closed subset of $X$. Assume that $(\bar{x}, \bar{v})$ with $\bar{x} \in \Omega$, $\bar{z} \in G(\bar{x})$, and $\bar{v}:=\bar{z}+\sum_{i=1}^{n} \alpha_{i}\left\|A_{i}(\bar{x})-a^{i}\right\|$ is a minimizer of problem (5.3) and that $G$ is Lipschitzian-like at $(\bar{x}, \bar{y})$. Assume that $\Theta$ is $S N C$ at $\mathbf{0}$ and the qualification condition

$$
y^{*} \in-N(\mathbf{0} ; \Theta) \cap H_{0}(\mathbf{k}), \mathbf{0} \in D_{M}^{*} F(\bar{x}, \bar{v})\left(y^{*}\right)+N(\bar{x} ; \Omega) \Rightarrow y^{*}=\mathbf{0}
$$

for some $\mathbf{k} \in \operatorname{dir}(\Theta)$ is fulfilled. Then, there is $y^{*} \in Y^{*}$ with $z^{*}(\mathbf{k})=1$ such that

$$
0 \in \partial G(\bar{x}, \bar{y})\left(y^{*}\right)+\sum_{i=1}^{n} \alpha_{i} A_{i}^{*} y^{*} T_{i}+N(\bar{x} ; \Omega)
$$

where

$$
T_{i} \in L(Z, Y), T_{i}\left(A_{i}(\bar{x})-a^{i}\right)=\left\|A_{i}(\bar{x})-a^{i}\right\|, \forall z \in Z:\|z\|-T_{i}(z) \in \Theta,(i=1, \ldots, n) .
$$

Proof. Because $X, Y, Z$ are reflexive Banach spaces, they are Asplund spaces. By Lemma 5.2, the vector norm $\|\cdot\| \mid \|$ is Lipschitz and thus the cost mapping $F$ is Lipschitz-like at $(\bar{x}, \bar{v})$. By Lemma 2.6, $F$ is PSNC at $(\bar{x}, \bar{v})$ and $D_{M}^{*} F(\bar{x}, \bar{v})(\mathbf{0})=\{\mathbf{0}\}$. Then from Theorem 4.2 we get the existence of $y^{*} \in Y^{*}$ with $y^{*}(\mathbf{k})=1$ and

$$
0 \in \partial F(\bar{x}, \bar{v})\left(y^{*}\right)+N(\bar{x}, \Omega) .
$$

The rule of sums for the Mordukhovich subdifferential yields the relation

$$
\partial F(\bar{x}, \bar{v})\left(y^{*}\right) \subseteq \partial G(\bar{x}, \bar{y})\left(y^{*}\right)+\sum_{i=1}^{n} \alpha_{i} \partial\left(y^{*}\left(\left\|A_{i}(\cdot)-a^{i}\right\|\right)\right)(\bar{x}) .
$$

Applying Theorem 5.1 and [16, Example 2.22], relation (5.5) implies 


$$
\begin{aligned}
\partial F(\bar{x}, \bar{v})\left(y^{*}\right) & \subseteq \partial G(\bar{x}, \bar{y})\left(y^{*}\right)+\sum_{i=1}^{n} \alpha_{i} \partial\left(y^{*}\left(\left\|A_{i}(\cdot)-a^{i}\right\| \mid\right)\right)(\bar{x}) \\
& =\partial G(\bar{x}, \bar{y})\left(y^{*}\right)+\sum_{i=1}^{n} \alpha_{i} y^{*}\left(\partial^{\leq}\left\|A_{i}(\cdot)-a^{i}\right\|(\bar{x})\right) \\
& =\partial G(\bar{x}, \bar{y})\left(y^{*}\right)+\left.\sum_{i=1}^{n} \alpha_{i} A_{i}^{*} y^{*}\left(\partial \leq\left\|u_{i}\right\| \|\right)\right|_{u_{i}=A_{i}(\bar{x})-a^{i}} \\
& =\partial G(\bar{x}, \bar{y})\left(y^{*}\right)+\left\{\sum_{i=1}^{n} \alpha_{i} A_{i}^{*} y^{*} T_{i} \mid T_{i} \in L(Y, Z), T_{i}\left(A_{i}(\bar{x})-a^{i}\right)=\right. \\
& \left.\left\|A_{i}(\bar{x})-a^{i}\right\|, \forall z \in Z:\|z\|-T_{i}(z) \in \Theta,(i=1, \ldots, n)\right\} .
\end{aligned}
$$

Then we get together with (5.4) the desired relation.

Remark 5.1. In [9] and [10], Lagrange multiplier rules are shown for weak Pareto minimizers. In comparison with the corresponding results in [9, Theorem 5.2] we show our results for Pareto minimizers and we do not need the assumption that int $\Theta \neq \emptyset$.

\section{Conclusions}

In this paper, we investigated optimality conditions for set-valued optimization problems based on the vector approach using a nonlinear scalarization method by means of the nonlinear functional (2.4) and concepts of generalized differentiation. In order to prove the optimality conditions for set-valued optimization problems based on the set approach, we will use our results shown for set-valued optimization problems based on the vector approach in a forthcoming paper. Furthermore, we will show relationships between solutions of set-valued optimization problems based on set approach and solutions based on vector approach that are useful in this context.

\section{Acknowledgment}

This research was supported by Alexander von Humboldt Foundation.

\section{REFERENCES}

[1] T.Q. Bao, B.S. Mordukhovich, Relative Pareto minimizers in multiobjective optimization: existence and optimality conditions, Math. Program. 122 (2010), 301-347.

[2] B.Q. Bao, B.S. Mordukhovich, Extended Pareto Optimality in Multiobjective Problem. In: Ansari QH, Yao J-C (eds.), Recent Advances in Vector Optimization, pp. 379-417. Springer, Berlin, 2011.

[3] T.Q. Bao, C. Tammer, Lagrange necessary conditions for Pareto minimizers in Asplund spaces and applications. Nonlin. Anal. 75 (2011) 1089-1103.

[4] T.Q. Bao, Subdifferential necessary conditions for extremal solutions to set-valued optimization problems with equilibrium constraints, Optimization 63 (2011), 181-205.

[5] T.Q. Bao, M. Hillmann, C. Tammer, Subdifferentials of nonlinear scalarization functionals and applications, J. Nonlin. Convex Anal. 18 (2017), 589-605.

[6] J.M. Borwein, Continuity and differentiability properties of convex operators, Proc. London Math. Soc. 44 (1982), $420-$ 444. 
[7] M. Durea, J. Dutta, Lagrange multipliers for Pareto minima in general Banach spaces, Pacific J. Optim. 4 (2008), $447-463$.

[8] M. Durea, R. Strugariu, On some Fermat rules for set-valued optimization problems, Optimization 60 (2011), $575-591$.

[9] M. Durea, C. Tammer, Fuzzy necessary optimality conditions for vector optimization problems, Optimization 58 (2009), 449-467.

[10] J. Dutta, C. Tammer, Lagrangian conditions for vector optimization in Banach spaces, Math. Method Oper. Res. 64 (2006), 521-540.

[11] G. Eichfelder, M. Pilecka, Set approach for set optimization with variable ordering structures Part I: Set relations and relationship to vector approach, J. Optim. Theory Appl. 171 (2016), 931-946.

[12] G. Eichfelder, M. Pilecka, Set approach for set optimization with variable ordering structures Part II: Scalarization approaches, J. Optim. Theory Appl. 171 (2016), 947-963.

[13] N.J. Huang, A.M. Rubinov, X.Q. Yang, Vector optimization problems with nonconvex preferences, J. Global Optim. 40 (2008), 765-777.

[14] C. Gerth (Tammer), P. Weidner, Nonconvex separation theorems and some applications in vector optimization, J. Optim. Theory Appl. 67 (1990), 297-320.

[15] A. Göpfert, H. Riahi, C. Tammer, C. Zălinescu, Variational Methods in Partially Ordered Spaces, New York, SpringerVerlag, 2003.

[16] J. Jahn, Vector Optimization, Theory, Applications and Extensions, 2nd edition, Heidelberg, Springer, 2011.

[17] A.A. Khan, C. Tammer, C. Zălinescu, Set-Valued Optimization, An Introduction with Applications, Heidelberg, Springer, 2015.

[18] E. Köbis, C. Tammer, J.-C. Yao, Optimality conditions for set-valued optimization problems based on set approach and applications in uncertain optimization, J. Nonlinear Convex Anal. 18 (2017), 1001-1014.

[19] D. Kuroiwa, On set-valued optimization, Proceedings of the Third World Congress of Nonlinear Analysts, Part 2 (Catania, 2000), Nonlinear Anal. 47 (2001), 1395-1400.

[20] B.S. Mordukhovich, Variational Analysis and Generalized Differentiation, I. Basic theory, Vol. 330, Berlin, Springer, 2006.

[21] B.S. Mordukhovich, Variational Analysis and Generalized Differentiation, II. Applications, Vol. 331, Berlin, Springer, 2006.

[22] C. Tammer, A generalization of Ekeland's variational principle, Optimization 5 (1992), 129-141.

[23] M. Valadier, Sous-différentiabilité de fonctions convexes à valeurs dans un espace vectoriel ordonné, Math. Scand. 30 (1972) 65-74.

[24] C. Tammer, C. Zălinescu, Lipschitz properties of the scalarization function and applications, Optimization 59 (2010), 305-319. 